\title{
On Extracting Physical Content from Asymptotically Flat Space-Time Metrics
}

\author{
C. Kozameh ${ }^{1}$, E. T. Newman ${ }^{2}$, G. Silva-Ortigoza ${ }^{3}$ \\ ${ }^{1} \mathrm{FaMaF}$, Univ. of Cordoba, \\ Cordoba, Argentina \\ ${ }^{2}$ Dept of Physics and Astronomy, \\ Univ. of Pittsburgh, \\ Pittsburgh, PA 15260, USA \\ ${ }^{3}$ Facultad de Ciencias Físico Matemáticas \\ de la Universidad Autónoma de Puebla, \\ Apartado Postal 1152, 72001, \\ Puebla, Pue., México
}

\subsection{2 .08}

\begin{abstract}
A major issue in general relativity, from its earliest days to the present, is how to extract physical information from any solution or class of solutions to the Einstein equations. Though certain information can be obtained for arbitrary solutions, e.g., via geodesic deviation, in general, because of the coordinate freedom, it is often hard or impossible to do. Most of the time information is found from special conditions, e.g., degenerate principle null vectors, weak fields close to Minkowski space (using coordinates close to Minkowski coordinates) or from solutions that have symmetries or approximate symmetries. In the present work we will be concerned with asymptotically flat space times where the approximate symmetry is the Bondi-Metzner-Sachs (BMS) group. For these spaces the Bondi four-momentum vector and its evolution, found from the Weyl tensor at infinity, describes the total energy-momentum of the interior source and the energy-momentum radiated. By generalizing the structures (shear-free null geodesic congruences) associated with the algebraically special metrics to asymptotically shear-free null geodesic congruences, which are available in all asymptotically flat space-times, we give kinematic meaning to the Bondi four-momentum. In other words we describe the Bondi vector and its evolution in terms of a center of mass position vector, its velocity and a spin-vector, all having clear geometric meaning. Among other items, from dynamic arguments, we define a unique (at our level of approximation) total angular momentum and extract its evolution equation in the form of a conservation law with an angular momentum flux.
\end{abstract}




\section{Introduction}

From the very earliest days of general relativity (GR) the issue of extracting the physical meaning or content in the solutions of the Einstein equations has been a difficult problem 1 and usually has been solved only in special cases or special situations, e.g., in the presence of symmetries or approximate (asymptotic) symmetries. Probably the best studied case is that of asymptotically flat space-times where an asymptotic symmetry exists, namely the BMS group, and where, at null infinity, the total energy-momentum of the interior source was identified as well as gravitational radiation i.e., energy and momentum loss. These identifications, in terms of the asymptotic components of the Weyl tensor, were made via group theoretical arguments combined with physical insight into the dynamics of the radiation process. However the problem of gravitational multipole moments, and in particular, the mass dipole and the angular momentum has proved to be difficult. At the present time, using symmetry arguments, there are several different proposed definitions [2, 3, 4, 5] of these quantities with little apparent use of dynamic considerations. (To our knowledge only in linear theory or in stationary cases has the issue of quadrupole moments been addressed.) In the present work, using largely a dynamic argument and verifying it by a symmetry consideration, we return to the issue of recognizing the mass-dipole moment and angular momentum hidden in the asymptotic Weyl tensor. From this dynamic argument the mass-dipole moment and angular momentum, using the Bondi energy-momentum four-vector, are found. Both kinematic expressions, as well dynamic equations (equations of motion), are determined.

In flat-space Maxwell theory the related issues are much simpler though some questions do remain. The charge, obtained by a 2-D surface integral of the asymptotic Maxwell field is a constant and lies in the scalar representation of the Lorentz group; the dipole moments (electric and magnetic) also obtained by 2-D surface integrals of the asymptotic Maxwell field (though the results depend on a time direction and the 2-surface chosen for the integral) also lie in a finite dimensional representation of the Lorentz group. For a static or stationary system it is easy to define the center of charge (so that the electric dipole moment associated with it vanishes) and a constant magnetic dipole moment. Recently it was shown[ 6 ] that for general asymptotically flat Maxwell fields (with non-vanishing total charge) one could find a unique complex worldline in complex Minkowski space, referred to as the complex center of charge, from which the dynamic electric and magnetic moments could be obtained.

It is this construction of the complex center of charge for the Maxwell field that we generalize to GR. This leads, in a very unorthodox manner, to a complex center of mass. The real part is identified with the usual center of mass while the imaginary part is the specific spin-angular momentum, i.e., the spin per unit mass. The argument leading to this complex world line is based largely on dynamics and analogies with electrodynamics and special solutions of GR, e.g., the algebraically special metrics and in particular, the charged spinning metric [7. It is applied to both the asymptotic vacuum Einstein equations and 
the Einstein-Maxwell equations. For ease of presentation most of the analysis will be for the vacuum case. The results for the Einstein-Maxwell case will be presented towards the end of Sec. III without detailed derivation.

In order to get a better perspective on this work and on our point of view, several comments might be of some use.

Both Maxwell theory and GR are considered to be fundamental physical theories [in addition to quantum theory which so far appears incompatible with GR] and thus in principle this construction is applicable to the gravitational field in the neighborhood of any massive body, from elementary particles to galaxies. Though GR is almost always applied to astrophysical situations, there is in principle no reason that it could not be applied at some level to laboratory masses or even to elementary particles. If the individual masses are sufficiently far apart we could even consider their gravitational fields at (relatively) large distances as being asymptotically flat and use the general theory of asymptotically flat space-times for their analysis. The theory of asymptotically flat space-times appears to be the best tool to define isolated bodies in GR and, as in Newton's theory, the notion of isolated bodies is an approximation of reality that is useful in general, and specifically for defining center of mass, intrinsic angular momentum, etc.

It is this point of view we adopt in this work. Though there is no rigorous way to make physical identification with the GR variables we will consider three very similar alternative identifications - some more intuitive than others - and see what are the theoretical consequences - and then argue for the last one. The idea is then to look at the dynamic predictions of each of the alternatives. At the linear level they all agree: it is in the consideration of the non-linear terms that we see the differences and see how one of the alternatives, probably the least intuitive one, comes closest to what we would physically expect.

The starting point for the discussion are the asymptotic Bianchi identities. We have assumed that the Einstein or the Einstein/Maxwell equations have been integrated in the asymptotic region using the standard Bondi-Sachs-NewmanPenrose asymptotic [8, 9, 10, 11] (peeling theorem) behavior. When the relevant integrations have been completed we are left with several evolution equations, namely the asymptotic Bianchi Identities (the Bondi supplementary conditions) and asymptotic Maxwell equations 12, that become our object for analysis and interpretation.

In Sec. II we review the relevant ideas about asymptotically flat space-times (Einstein or Einstein/Maxwell) at null infinity 12. More specifically we discuss the structure of null infinity (and its complexification), i.e., Penrose's $\mathfrak{I}^{+}$. We then review a variety of subjects: e.g., what 'lives' on $\mathfrak{I}^{+}$, the asymptotic Weyl tensor with the Bianchi Identities and the Bondi shear, other related structures as cuts of $\mathfrak{I}^{+}$, past-light-cones and angle fields. The spherical harmonic decomposition of the various fields is then described. In this context we discuss the Bondi four-momentum and the energy/momentum loss theorem.

In Sec. III, we examine three alternative suggestions for extracting physical information from the Weyl tensor components in the asymptotic Bianchi Identities. A central structure in this extraction process comes from the dynamics 
associated with the Bondi four-momentum. More specifically we want first to identify the mass dipole (or center of mass) and angular momentum. Then, second, we want to establish dynamical laws for these quantities, i.e., we want equations of motion for the center of mass and evolution equations - with fluxes - for the angular momentum. They come directly from the energy-momentum loss equations and a certain reality condition. These dynamical laws are given at future null infinity and therefore are intended to apply to the entire isolated system. Interactions between different bodies can not be considered in this framework.

The first and most 'obvious' or intuitive model for the selection for the mass dipole and angular momentum turns out to lack certain essential dynamic features and has little or no geometric meaning. We consider the first (and second) approaches as 'toy' models, introduced in order motivate and clarify the third and primary model. The second and the third approaches which are based on specific choices of complex slicing of future null infinity, referred to as (complex) cut functions, lead to very similar dynamics. The nod goes to the third choice, it being more 'natural' in its similarity or analogy to the algebraically special metrics and to its unity and geometric attractiveness. They are both based on a geometric construction of complex cut functions. One of them, the second, is more appropriate to flat space, the other (which does reduce to the second in flat space) is far better suited to the general asymptotically flat case. In the later case we give an extended description of the predictions and dynamic results including the results for the Einstein-Maxwell equations. These results include kinematic expressions for the Bondi mass and momentum, a center of mass and its equations of motion and a definition of angular momentum that includes both spin and orbital terms with a conservation law with an angular momentum flux.

In Sec. IV we analyze the invariance, under the BMS group, of the third approach to this method of assigning physical meaning to the variables. We briefly discuss the representation theory of the Lorentz group due to Gelfand, Graev and Vilinkin 13, 14, and in particular apply their description of the invariant finite dimensional subspaces of the infinite dimensional representations, to our physical assignments. As a simple example, we apply the representation theory to the Bondi energy-momentum four-vector and show that indeed it is a Lorentzian four-vector. The details are given in appendix 7.2.

In the discussion section, Sec. V, we summarize and discuss our results, including the results for the Einstein-Maxwell case. We also speculate on possible consequences of these results. One of our more interesting results worth speculating on - is that a simple geometric condition on the EinsteinMaxwell fields leads to the Dirac value of the gyromagnetic ratio.

Our method for the study of the physical identifications is based on the use of spherical harmonic expansions so that the coefficients in these expansions become identified with physical objects. It is clear from the non-linearity of the theory that it is impossible to work with the exact infinite expansions and, thus, we must work with a truncated series. Specifically we expand everything up to and including the $l=2$ harmonics and then include only $2^{\text {nd }}$ order products. 
(In one place, where the result is physically so attractive, we have included a $3^{\text {rd }}$ order term.) Almost all of the non-linear terms in our results arise from the (frequent) use of Clebsch-Gordon expansions of spherical harmonic products.

\section{Preliminaries}

\section{$2.1 \quad$ Null Infinity}

After integrating [8, 9, 10, 11, 12] the Einstein (or Einstein-Maxwell) equations along null surfaces in the spin-coefficient formalism for large affine parameter, $r$, we are left, in the limit of large $r$ with several evolution equations [12] (for the asymptotic Weyl tensor components) that are referred to as the "asymptotic Bianchi identities". (These equations are analogous to the remaining Maxwell equations 12 after all the radial integrations have been performed.) This limit of large $r$ has been formalized by Penrose into the idea of the future null boundary of space-time, a null three-surface, and referred to as $\mathfrak{I}^{+}$. Our remaining equations are thus a set of differential equations on $\mathfrak{I}^{+}$. This null boundary has the topology of $S^{2} \times R$ and an assignment of (Bondi) coordinates $(u, \zeta, \bar{\zeta})$, with $u$ on the $R$ part and $(\zeta, \bar{\zeta})$ as complex stereographic coordinates on the $S^{2}$. The freedom in the choice of these Bondi coordinates, known as the BMS group is geometrically the asymptotic symmetry group. The BMS group is composed of two parts, the supertranslations

$$
\begin{aligned}
\widehat{u} & =u+\alpha(\zeta, \bar{\zeta}) \\
(\widehat{\zeta}, \overline{\widehat{\zeta}}) & =(\zeta, \bar{\zeta})
\end{aligned}
$$

with $\alpha(\zeta, \bar{\zeta})$ an arbitrary smooth function on the sphere and the Lorentz transformations 8 , 9, 15, 16, 14, given by

$$
\begin{aligned}
\widehat{u} & =K u \\
K & =\frac{1+\zeta \bar{\zeta}}{(a \zeta+b)(\bar{a} \bar{\zeta}+\bar{b})+(c \zeta+d)(\bar{c} \bar{\zeta}+\bar{d})} \\
\widehat{\zeta} & =\frac{a \zeta+b}{c \zeta+d} ; \quad a d-b c=1 .
\end{aligned}
$$

Though we are dealing with real space-times and $\mathfrak{I}^{+}$is a real three-surface, since we assume that all the relevant functions are analytic, it is useful to allow the $u$ to take on complex values close to the real and to allow $\bar{\zeta}$ to deviate slightly from the complex conjugate of $\zeta$.

In addition to the Bondi coordinates we also have a null tetrad $\left(l^{a}, n^{a}, m^{a}, \bar{m}^{a}\right)$ that is associated with the Bondi coordinates (and does change with a BMS coordinate transformation) where $n$ is tangent to the null generators of $\mathfrak{I}^{+}, m^{a}$ and $\bar{m}^{a}$ are tangent to the $u=$ constant slices' of $\mathfrak{I}^{+}$while $l$ is the null vector normal to the slices pointing into the space-time along the null surface $u=$ constant. 


\section{$2.2 \quad$ Further Structures}

There are several important structures associated with $\mathfrak{I}^{+}$that we now describe.

- In addition to the Bondi 'slicing' of $\mathfrak{I}^{+}$given by the 'cuts', $u=$ constant, one can take an arbitrary real one-parameter, $s$, family of slices given by real cut functions,

$$
u=G(s, \zeta, \bar{\zeta}),
$$

or their generalization to analytic complex cut functions

$$
u=X(\tau, \zeta, \bar{\zeta})
$$

with inverse functions

$$
\tau=T(u, \zeta, \bar{\zeta})
$$

where the complex $\tau$ must be able to be chosen so that $u$ is real. The freedom in the choice of the parameter $\tau: \widehat{\tau}=F(\tau)$ with $F$ analytic, will be used later to normalize a physical variable.

- At every point of $\mathfrak{I}^{+}$there is the past light-cone of rays going back into the interior. The directions are labeled by the complex stereographic angle $(L, \bar{L})$ with the zero value taken along $l$ and the infinity along $n$. Any complex angle field $L(\zeta, \bar{\zeta})$, i.e., a stereographic angle given for each point on $\mathfrak{I}^{+}$, can be expressed [17] in terms of a complex cut function, restricted to real $u^{\prime} \mathrm{s}$ by

$$
\begin{aligned}
L(u, \zeta, \bar{\zeta}) & =\varlimsup_{(\tau)} X(\tau, \zeta, \bar{\zeta}) \\
\tau & =T(u, \zeta, \bar{\zeta}) .
\end{aligned}
$$

The subscript $(\tau)$ means the application of $\partial$ holding $\tau$ constant.

- We will have considerable use for the local Lorentz transformation (null rotations) at each point of $\mathfrak{I}^{+}$parametrized by the arbitrary angle field

$$
L=L(u, \zeta, \bar{\zeta})
$$

that preserve the vector $n$, namely

$$
\begin{aligned}
l^{*} & =l+L \bar{m}+\bar{L} m+L \bar{L} n \\
m^{*} & =m+L n \\
n^{*} & =n
\end{aligned}
$$

For each point on $\mathfrak{I}^{+}$, the null vector $l^{*}$ determines a null geodesic extending backwards into the space-time so that the field of $l^{* \prime}$ s determines a null geodesic congruence of the space-time. 


\subsection{What lives on $\mathfrak{I}^{+}$?}

The following functions are defined[12] on $\mathfrak{I}^{+}$;

- $\sigma=\sigma(u, \zeta, \bar{\zeta})$, the asymptotic shear of the null geodesic congruence with Bondi tangent vector $l$.

- $\psi_{1}^{0}(u, \zeta, \bar{\zeta})$ and $\psi_{2}^{0}(u, \zeta, \bar{\zeta})$ are the leading terms of two tetrad components of the Weyl tensor, $\psi_{1}=\psi_{1}^{0} / r^{4}+\ldots$ and $\psi_{2}=\psi_{2}^{0} / r^{3}+\ldots$

with

$$
\begin{aligned}
& \psi_{1}=-C_{a b c d} l^{a} m^{b} l^{c} m^{d} \\
& \psi_{2}=-C_{a b c d} \bar{m}^{a} n^{b} l^{c} m^{d}
\end{aligned}
$$

Under the tetrad transformation, Eq.(7), the Weyl components $\psi_{1}^{0}$ and $\psi_{2}^{0}$ transform as

$$
\begin{aligned}
& \psi_{1}^{0 *}=\psi_{1}^{0}-3 L \psi_{2}^{0}+3 L^{2} ð(\bar{\sigma})^{\cdot}+L^{3} \bar{\sigma} \\
& \psi_{2}^{0 *}=\psi_{2}^{0}-2 L ð(\bar{\sigma})^{\cdot}-L^{2} \bar{\sigma} \cdot
\end{aligned}
$$

The $\psi_{1}^{0}$ and $\psi_{2}^{0}$ satisfy the asymptotic Bianchi Identities

$$
\begin{aligned}
\left(\psi_{1}^{0}\right)^{\cdot} & =-ð \psi_{2}^{0}+2 \sigma ð(\bar{\sigma})^{\cdot} \\
\left(\psi_{2}^{0}\right)^{\cdot} & =-ð^{2}(\bar{\sigma})^{\cdot}-\sigma(\bar{\sigma})^{\cdot} \\
\psi_{2}^{0}-\bar{\psi}_{2}^{0} & =\bar{\varpi}^{2} \sigma-ठ^{2} \bar{\sigma}+(\sigma)^{\cdot} \bar{\sigma}-(\bar{\sigma})^{\cdot} \sigma .
\end{aligned}
$$

The last two equations can be rewritten in terms of the 'mass aspect', $\Psi$, as

$$
\begin{aligned}
\Psi & =\sigma^{\cdot} \bar{\sigma} \\
\Psi & =\bar{\Psi}=\psi_{2}^{0}+\partial^{2} \bar{\sigma}+\sigma(\bar{\sigma})^{.} .
\end{aligned}
$$

When a Maxwell field is present, these equations become modified and the Maxwell equations must be included[12] :

$$
\begin{gathered}
\left(\psi_{1}^{0}\right)^{\cdot}=-ð \psi_{2}^{0}+2 \sigma ð(\bar{\sigma})^{\cdot}+2 k \phi_{1}^{0} \bar{\phi}_{2}^{0} \\
\left(\psi_{2}^{0}\right)^{\cdot}=-\partial^{2}(\bar{\sigma})^{\cdot}-\sigma(\bar{\sigma})^{\cdot}+k \phi_{2}^{0} \bar{\phi}_{2}^{0} \\
\bar{\Psi}=\Psi=\psi_{2}^{0}+\partial^{2} \bar{\sigma}+\sigma(\bar{\sigma})^{\cdot}, \\
k=2 G c^{-4} \\
\left(\phi_{0}^{0}\right)^{\cdot}+ð \phi_{1}^{0}-\sigma \phi_{2}^{0}=0, \\
\left(\phi_{1}^{0}\right)^{\cdot}+ð \phi_{2}^{0}=0,
\end{gathered}
$$




\subsection{Asymptotically Shear-free Null Geodesic Congruences}

- If the angle field $L(u, \zeta, \bar{\zeta})$ satisfies the differential equation 18, 17,

$$
ð L+L L=\sigma(u, \zeta, \bar{\zeta})
$$

the null geodesic congruence determined by the null vector field given by Eq. (17) is asymptotically shear-free. It has been shown earlier [17 that solutions to Eq.(21) that are regular on $\mathfrak{I}^{+}$, i.e., have no infinities, are given by the following construction:

$L(u, \zeta, \bar{\zeta})$ is given parametrically by

$$
\begin{aligned}
L(u, \zeta, \bar{\zeta}) & =\varlimsup_{(\tau)} X(\tau, \zeta, \bar{\zeta}) \\
u & =X(\tau, \zeta, \bar{\zeta})
\end{aligned}
$$

where $X(\tau, \zeta, \bar{\zeta})$ is found by first solving the 'good cut' equation[19],

$$
\check{\partial}^{2} Z=\sigma(Z, \zeta, \bar{\zeta})
$$

whose solutions are known to depend on four arbitrary complex parameters, $z^{a}$, i.e., $Z=Z\left(z^{a}, \zeta, \bar{\zeta}\right)$. By choosing an arbitrary world-line in the parameter space, $\left(H\right.$-space[19]), i.e., $z^{a}=\xi^{a}(\tau), X(\tau, \zeta, \bar{\zeta})$ is determined by

$$
u=X(\tau, \zeta, \bar{\zeta})=Z\left(\xi^{a}(\tau), \zeta, \bar{\zeta}\right)
$$

We thus have that every regular solution to the asymptotically shear-free condition, Eq.( 21), is determined by an arbitrary complex world-line in a fourcomplex dimensional parameter space. The freedom, mentioned earlier, in the choice of $\tau$ is used later to give a normalization to $v^{a}(\tau) \equiv \partial_{\tau} \xi^{a}$.

We could reverse the procedure just described and assume that $u=X(\tau, \zeta, \bar{\zeta})$ was given rather than $\sigma(u, \zeta, \bar{\zeta})$. Then $\sigma(u, \zeta, \bar{\zeta})$ and $L(u, \zeta, \bar{\zeta})$ could be determined parametrically by

$$
\begin{aligned}
u & =X(\tau, \zeta, \bar{\zeta}) \\
L(u, \zeta, \bar{\zeta}) & =\partial_{(\tau)} X(\tau, \zeta, \bar{\zeta}) \\
\sigma(u, \zeta, \bar{\zeta}) & =ð_{(\tau)}^{2} X(\tau, \zeta, \bar{\zeta})
\end{aligned}
$$

or more explicitly by the harmonic series,

$$
\begin{aligned}
u & =X(\tau, \zeta, \bar{\zeta})=\frac{1}{\sqrt{2}} \xi^{0}(\tau)-\frac{1}{2} \xi^{i}(\tau) Y_{1 i}^{0}(\zeta, \bar{\zeta})+\xi^{i j}(\tau) Y_{2 i j}^{0}(\zeta, \bar{\zeta})+\ldots \\
L(u, \zeta, \bar{\zeta}) & =\xi^{i}(\tau) Y_{1 i}^{1}(\zeta, \bar{\zeta})-6 \xi^{i j}(\tau) Y_{2 i j}^{1}(\zeta, \bar{\zeta})+\ldots \\
\sigma(u, \zeta, \bar{\zeta}) & =24 \xi^{i j}(\tau) Y_{2 i j}^{2}+\ldots
\end{aligned}
$$

with $\xi^{a}=\left(\xi^{0}(\tau), \xi^{i}(\tau)\right)$. The complex parameter $\tau$ is given by $\tau=T(u, \zeta, \bar{\zeta})$, Eq.(66). Note the important point that when $\tau$ is replaced by $T$, the spherical 
harmonic decomposition of those variables becomes non-trivial since it involves products of different spherical harmonics.

In the special case of flat-space, (with $\sigma(u, \zeta, \bar{\zeta})=0$ ), the asymptotically shear-free congruences become shear-free congruences and the regular solutions to Eq.(21), given parametrically, become

$$
\begin{aligned}
u & =X(\tau, \zeta, \bar{\zeta})=\xi^{a}(\tau) \widehat{l}_{a}(\zeta, \bar{\zeta})=\frac{1}{\sqrt{2}} \xi^{0}(\tau)-\frac{1}{2} \xi^{i}(\tau) Y_{1 i}^{0}(\zeta, \bar{\zeta}) \\
L(u, \zeta, \bar{\zeta}) & =ð X(\tau, \zeta, \bar{\zeta})=\xi^{a}(\tau) m_{a}(\zeta, \bar{\zeta}) \\
\widehat{l}_{a}(\zeta, \bar{\zeta}) & =\frac{\sqrt{2}}{2}\left(1, \frac{\zeta+\bar{\zeta}}{1+\zeta \bar{\zeta}},-i \frac{\zeta-\bar{\zeta}}{1+\zeta \bar{\zeta}}, \frac{-1+\zeta \bar{\zeta}}{1+\zeta \bar{\zeta}}\right), \\
\widehat{m}_{a}(\zeta, \bar{\zeta}) & =ð \widehat{l}_{a}(\zeta, \bar{\zeta})=\frac{\sqrt{2}}{2 P}\left(0,1-\bar{\zeta}^{2},-i\left(1+\bar{\zeta}^{2}\right), 2 \bar{\zeta}\right),
\end{aligned}
$$

with $\widehat{l}_{a}(\zeta, \bar{\zeta})$ a flat-space null vector on the 'light-cone' composed of the $l=0,1$ harmonics. In other words the regular flat-space shear-free null geodesic congruences are determined by an analytic complex curve in complex Minkowski space. One can reinterpret the Minkowski space curve as a curve in the space of asymptotic complex Poincare translations. The advantage of this latter interpretation is that it applies just as well to the asymptotically flat space-times.

\subsection{Spherical Harmonic Decomposition}

All the functions on $\mathfrak{I}^{+}$that we are dealing with have a spin weight $(s)$ and most have a definite conformal weight $(w)$. They thus can be expanded in the spin-weighted tensor harmonics- 20. Denoting the spin and conformal weights by $(s, w)$ as a subscript e.g., $W_{(s, w)}$, we have

$$
\begin{gathered}
u=X \equiv X_{(0,1)}=\frac{1}{\sqrt{2}} \xi^{0}(\tau)-\frac{1}{2} \xi^{i}(\tau) Y_{1 i}^{0}(\zeta, \bar{\zeta})+\xi^{i j}(\tau) Y_{2 i j}^{0}(\zeta, \bar{\zeta})+\ldots \\
\sigma \equiv \sigma_{(2,-2)}=24 \xi^{i j}(\tau) Y_{2 i j}^{2}+\ldots \\
L \equiv L_{(1, \times)}=\xi^{i}(\tau) Y_{1 i}^{1}(\zeta, \bar{\zeta})-6 \xi^{i j}(\tau) Y_{2 i j}^{1}(\zeta, \bar{\zeta})+\ldots \\
\psi_{2}^{0} \equiv \psi_{2(0,-3)}^{0}=\Upsilon+\psi_{2 i}^{0} Y_{1 i}^{0}+\psi_{2 i j}^{0} Y_{2 i j}^{0}+\ldots \\
\psi_{1}^{0} \equiv \psi_{1(1,-3)}^{0}=\psi_{1 i}^{0} Y_{1 i}^{1}+\psi_{1 i j}^{0} Y_{2 i j}^{1}+\ldots \\
\Psi \equiv \Psi_{(0,-3)}=\Psi^{0}+\Psi^{i} Y_{1 i}^{0}+\Psi^{i j} Y_{2 i j}^{0}+\ldots \\
\phi_{0}^{0}=\phi_{0 i}^{0} Y_{1 i}^{1}+\phi_{0 i j}^{0} Y_{2 i j}^{1}+\ldots \\
\phi_{1}^{0}=Q+\phi_{1 i}^{0} Y_{1 i}^{0}+\phi_{1 i j}^{0} Y_{2 i j}^{0}+\ldots \\
\phi_{2}^{0}=\phi_{2 i}^{0} Y_{1 i}^{-1}+\phi_{2 i j}^{0} Y_{2 i j}^{-1}+\ldots
\end{gathered}
$$


The basic idea is to try to give physical meaning or significance to the harmonic coefficients. An important well known example of this is Bondi's identification of the $l=(0,1)$ parts of the mass aspect with the total energy-momentum four-vector of the interior sources, $\left(M c, P^{i}\right)$ which is explicitly given by

$$
\Psi=-\frac{2 \sqrt{2} G}{c^{2}} M-\frac{6 G}{c^{3}} P^{i} Y_{1 i}^{0}+\ldots
$$

From the $l=0$ harmonic coefficient in Eq. (13) one obtains the Bondi mass loss equation which allows us to identify the coefficient $\xi^{i j}(u)$ as proportional to the $2^{\text {nd }}$ derivative of the mass quadrupole.

A few other identifications coming from Maxwell theory are: $Q$ is the Coulomb charge, while $\phi_{0 i}^{0}$ is proportional to the complex electromagnetic dipole moment (electric $+i$ magnetic), $\phi_{2 i}^{0}$ is proportional to the $2^{\text {nd }}$ time derivative of $\phi_{0 i}^{0}$ while $\phi_{2 i j}^{0}$ is proportional to the $3^{\text {rd }}$ derivative of the quadrupole moment.

Very roughly speaking we make the approximate identification of $\psi_{1 i}^{0}$, (which is complex) with the complex gravitational dipole moment, i.e., with the mass dipole plus ' $i$ ' times the angular momentum.

\section{$3 \quad$ Identifications}

Our starting point for the physical identifications is Eq.(10)

$$
\left(\psi_{1}^{0}\right)^{\cdot}=-ð \psi_{2}^{0}+2 \sigma \partial(\bar{\sigma})^{\cdot}
$$

and the spherical harmonic decomposition. We noted that the $l=1$ part of $\psi_{2}^{0}$ was proportional to the Bondi three-momentum, $\vec{P}$. The linearized version of Eq. (46) leads to the fact that the $l=1$ part of $\left(\psi_{1 i}^{0}\right)^{\cdot}$ is also proportional to $\vec{P}$. This in turn suggests that $\psi_{1 i}^{0}$ itself should be, at least in the linearized version, proportional to the mass-dipole, $M \vec{X}$, where $\vec{X}$ should be identified with the position vector of center of mass so that $M \vec{X} \cdot=\vec{P}$.

This is our fundamental observation. It will be analyzed and generalized.

We begin by postulating three different models or methods for the identification. Though we have one method (the third) that we consider to be fundamental and correct, nevertheless we felt that at least a few others should be explored to see if they would be reasonable choices. Our criteria for selecting a model are: (i) it should predict already known laws or reasonable new laws, (ii) it should have a clear geometric foundation and have a logical consistency and (iii) it should agree with special cases, mainly the algebraically special metrics or analogies with flat-space Maxwell theory.

The first (naive) approach is to simply assume that the Bondi 3-momentum is given by $\vec{P}=M \vec{X} \cdot=M \vec{V}$. The second approach is based on the flat-space transformation (translation) properties of dipoles, i.e., transforming to the center of mass or charge, while the third approach generalizes this to asymptotically flat space-times. The first two do not satisfy our three criteria while the third 
one does. We nevertheless felt it was worthwhile to see how the increasing sophistication of the methods led to improved results.

Remark 1. We have (for notational simplicity) totally abused standard notation. We allow the indices $(i, j, k, l \ldots)$, which are Euclidean, to be raised and lowered with impunity and allow repeated indices, e.g., $v^{k} \xi^{i k}$, to indicate summation.

Remark 2. Though $u$ is the conventional Bondi time, it is more appropriate to use $w=\sqrt{2} u$, it being the retarded time. Derivatives with respect to $u$ are denoted by dot, $\left({ }^{(}\right)$, while $w$ derivatives are given by a prime, $\left({ }^{\prime}\right)$, i.e., $\left({ }^{\cdot}\right)=\sqrt{2}\left({ }^{\prime}\right)$.

Claim A very important computational issue is to find the inversion of Eq.(36) ), i.e., to find $\tau=T(u, \zeta, \bar{\zeta})$. The reason for its importance is that we must be able to explicitly eliminate the $\tau$ and replace it by the Bondi $u$ (or $w$ ) in the parametric expressions for $L(u, \zeta, \bar{\zeta})$, i.e., in the expression for the complex world-line $\xi^{a}(\tau)$. The approximate inversion (linear) is given [21]by

$$
\tau=T(u, \zeta, \bar{\zeta})=w+\frac{\sqrt{2}}{2} \xi^{i}(w) Y_{1 i}^{0}(\zeta, \bar{\zeta})-\sqrt{2} \xi^{i j}(w) Y_{1 i j}^{0}(\zeta, \bar{\zeta})+\ldots
$$

\subsection{The First Identification Method}

For our first (toy) model, we assume that the Bondi momentum (at least at linear order) has the standard kinematic form, namely

$$
\vec{P}=M \vec{V}
$$

and try to find the consequences. From our perspective this already is unsatisfactory, since we would like this kinematic expression to follow directly from the Einstein equations rather than to assume it. In fact, in the second and third models this relationship is a derived result. Nevertheless from a heuristic point of view we believe it worthwhile to show the line of reasoning - in this simpler case, - before is used later for the preferred models. In addition, roughly speaking, from this first model we can see what consequences can be anticipated.

Thus from the above argument, we take $\psi_{1 i}^{0}$ to have the form

$$
\psi_{1 i}^{0}=\alpha M \lambda_{i}
$$

where $\alpha$ is a constant to be determined, $M$ is the Bondi mass and $\lambda_{i}$ is a complex three-vector in some unknown space (hopefully) to be determined. The real part of $\lambda_{i}$ is tentatively associated with the 'center of mass position' and its derivative with the 'center of mass' velocity, i.e., $\lambda_{R i}^{\prime}=v_{i} \Rightarrow \vec{V}$.

Using the harmonic expansions, Eqs.(39), (40) and Clebsch-Gordon products, [see appendix C], Eq.(46) becomes

$$
\psi_{2 k}^{0}=\frac{\sqrt{2}}{2 c} \psi_{1 k}^{0 \prime}-i \frac{36 \cdot 64}{5 c} \bar{\xi}^{m j \prime} \xi^{l m} \epsilon_{l j k} .
$$


while the expansion of the mass aspect, Eq.(41), is

$$
\Psi=\Upsilon(w)+\frac{16 \cdot 36 \sqrt{2}}{5} \xi^{i j} \bar{\xi}^{i j \prime}+\left(\psi_{2 k}^{0}+\frac{i(32)(36)}{5} \xi^{l j} \bar{\xi}^{i j \prime} \epsilon_{i l k}\right) Y_{1 k}^{0}+\ldots
$$

Using Eqs.(??), (50) and (49) we identify, from Eq.(45), the mass and threemomentum

$$
\begin{aligned}
M & =-\frac{c^{2}}{2 \sqrt{2} G} \Upsilon-\frac{288}{5} \frac{c}{G} \xi^{i j} \bar{\xi}^{i j \prime} \\
P^{k} & =M \lambda_{k}^{\prime}+i \frac{192}{5 G} c^{3} \bar{\xi}^{j m \prime} \xi^{l m} \epsilon_{l j k} \\
\alpha & =-\frac{12 G}{\sqrt{2} c^{2}} .
\end{aligned}
$$

Writing the variables as

$$
\begin{aligned}
\Upsilon & =\Upsilon_{R}+i \Upsilon \\
\lambda_{k} & =\lambda_{R k}+i \lambda_{I k} \\
\xi^{l m} & =\xi_{R}^{l m}+i \xi_{R}^{l m} \\
\xi^{l m \prime} & \equiv v^{l m}=v_{R}^{l m}+i v_{R}^{l m}
\end{aligned}
$$

the reality conditions, Eq.(14), becomes

$$
\begin{aligned}
M & =-\frac{c^{2}}{2 \sqrt{2} G} \Upsilon_{R}-\frac{36 \cdot 8}{5} \frac{c}{G}\left(\xi_{R}^{i j} v_{R}^{i j}+\xi_{I}^{i j} v_{I}^{i j}\right) \\
M & =M_{0}-\frac{36 \cdot 4}{5} \frac{c}{G}\left(\xi_{R}^{i j} \xi_{R}^{i j}+\xi_{I}^{i j} \xi_{I}^{i j}\right)^{\prime} \\
\Upsilon_{I} & =\frac{(24)^{2} \sqrt{2}}{5 c}\left(\xi_{R}^{i j} v_{I}^{i j}-\xi_{I}^{i j} v_{R}^{i j}\right) \\
P^{k} & =M \lambda_{R k}^{\prime}-\frac{(24)^{2} c^{2}}{5 G}\left(\xi_{I}^{i l} v_{R}^{i j}-\xi_{R}^{i l} v_{I}^{i j}\right) \epsilon_{l j k} \\
M \lambda_{I k}^{\prime} & =-\frac{(24)^{2} c^{2}}{5 G}\left(\xi_{R}^{i l} v_{R}^{i j}+\xi_{I}^{i l} v_{I}^{i j}\right) \epsilon_{l j k}
\end{aligned}
$$

Finally, Eq.(11),

$$
\left(\psi_{2}^{0}\right)^{\cdot}=-\check{\partial}^{2}(\bar{\sigma})^{\cdot}-\sigma(\bar{\sigma})^{\cdot \cdot},
$$

after using the Clebsch-Gordon expansion for $\sigma(\bar{\sigma})^{*}$, yields for the $l=0,1$ terms, the mass and momentum loss expressions,

$$
\begin{aligned}
& M^{\prime}=-\frac{288 c}{5 G}\left(v_{R}^{i j} v_{R}^{i j}+v_{I}^{i j} v_{I}^{i j}\right) \\
& P^{k \prime}=F^{k} \equiv \frac{192 c^{2}}{5 G}\left(v_{I}^{l j} v_{R}^{i j}-v_{R}^{l j} v_{I}^{i j}\right) \epsilon_{i l k} .
\end{aligned}
$$


There are now two identifications that can be made immediately from these expressions. From the quadrupole energy loss relations 25]

$$
M^{\prime}=-\frac{G}{5 c^{7}}\left(Q_{\text {Mass }}^{i j \prime \prime \prime} Q_{\text {Mass }}^{i j \prime \prime \prime}+Q_{\text {Spin }}^{i j \prime \prime \prime} Q_{\text {Spin }}^{i j \prime \prime \prime}\right)
$$

we can relate $\xi^{i j}$ to the mass and spin quadrupole moments by

$$
\xi^{i j}=\left(\xi_{R}^{i j}+i \xi_{I}^{i j}\right)=\frac{G}{12 \sqrt{2} c^{4}}\left(Q_{\text {Mass }}^{i j \prime \prime}+i Q_{\text {Spin }}^{i j \prime \prime}\right) .
$$

This identification applies to all three models.

Furthermore, we can substitute the expression for $\vec{P}$, i.e., Eq.(54), into the momentum loss equation, Eq.(57), obtaining equations of motion for the 'position' vector $\lambda_{R}^{i}$,

$$
M \lambda_{R k}^{\prime \prime}-\frac{(24)^{2} c^{2}}{5 G}\left(\xi_{I}^{m l} v_{R}^{m i}-\xi_{R}^{m l} v_{I}^{m i}\right)^{\prime} \epsilon_{l i k}=\frac{192 c^{2}}{5 G}\left(v_{I}^{l j} v_{R}^{i j}-v_{R}^{l j} v_{I}^{i j}\right) \epsilon_{i l k} .
$$

This can be interpreted as Newton's 2nd law, with the second term on the left coming from gravitational radiation reaction and the right-side from the 'rocket' or recoil force due to momentum loss.

For the last identification in this model we consider Eq.(55). From the analogy with the rotating mass solution, (with and without charge [7), and results, which will be described later, we identify

$$
S_{k}=M c \lambda_{I k}
$$

as the spin angular momentum so that Eq.(55) becomes the angular momentum loss equation,

$$
S_{k}^{\prime}=-\frac{(24)^{2} c^{3}}{5 G}\left(\xi_{R}^{i l} v_{R}^{i j}+\xi_{I}^{i l} v_{I}^{i j}\right) \epsilon_{l j k} .
$$

Summarizing the results for this physical model; we have assumed that the (complex) $l=1$ part of $\psi_{1 k}^{0}$ was proportional to the mass dipole moment and the spin angular momentum. The Bianchi identity Eq.(46) allowed us to find a kinematic expression for the Bondi linear momentum in terms of the mass dipole moment (or center of mass) while the Bianchi identity, Eq.(11), yielded the equations of motion. The $l=1$ reality condition became the angular momentum loss equation.

Though this model does lead to physical identifications that are quite reasonable, it nevertheless contains several very unsatisfactory features. First of all there is no hint of a geometric meaning for the 'position' vector $\lambda_{k}$. What space is it in? It was pulled out of thin air, just so that the momentum could be written as $\vec{P}=M \vec{V}$. The constant $\alpha$ was arbitrary until determined by the kinematic meaning of $\vec{P}$. A second deficiency is that the angular momentum is given only by the spin without any suggestion of an orbital part. Lastly, this identification is at total odds with the identifications obtained for the algebraically special metrics [26, 28, 27, 21] or with the analogous flat space Maxwell identification with the center of charge $[$.

We will see that the third model does correct these deficiencies. 


\subsection{The Second Identification Method}

Our second and third identification models are based on complex curves, in the second model these curves are associated with flat-space shear-free null geodesic congruences while in the third model they are associated with the asymptotically shear-free null geodesic congruences of the relevant space-time. These curves, as we mentioned earlier, are geometrically well defined as complex world-lines in the space of complex Poincare translations at $\mathfrak{I}^{+}$. The ideas involved are not obvious and do lie outside of the usual default understandings and thus it takes a bit of 'getting used to'. Basically, the idea is to generalize the standard action of moving the origin of coordinates to a new point, (the center of mass), so that the mass dipole vanishes for that origin. The generalization consists of making these transformations (translations) complex and moving to the complex center of mass, so that not only does the mass dipole vanish there, but so does the angular momentum. This type of procedure, which worked perfectly, in linearized gravity and Maxwell theory [22, 23] must now be applied to asymptotically flat space-times.

We begin with the observation that most attempts [2] at defining the angular momentum have the imaginary part of the $l=1$ harmonic of $\psi_{1}^{0}$ as part of the definition.

Remark 3. Most of the angular momentum definitions based on symmetry arguments start with the $l=1$ part of $\psi_{1}^{0}$ and augment it with terms quadratic in the shear and its derivatives. Later in this section we will return to the issue of these quadratic terms. See Appendix 7.4.

The central part of our argument is to use the $l=1$ part of the null rotated Weyl component, $\psi_{1}^{0 *}$, from Eq.(8), i.e.,

$$
\psi_{1}^{0 *}=\psi_{1}^{0}-3 L \psi_{2}^{0}+3 L^{2} \psi_{3}^{0}-L^{3} \psi_{4}^{0}
$$

as the transformed (translated) complex dipole moment and then set the $l=1$ part of $\psi_{1}^{0 *}$ to zero to (partially) determine the $L$. (In flat space this type of operation really moves the origin of Bondi light-cones to a new complex origin in complex Minkowski space so that the center of charge and the magnetic dipole both vanish.) In other words, we will set $\left.\psi_{1}^{0 *}\right|_{l=1}=0$ so that the $l=1$ part of $\psi_{1}^{0}$ is given by

$$
\left.\psi_{1}^{0}\right|_{l=1}=\left.\left.\left(3 L \psi_{2}^{0}-3 L^{2} \psi_{3}^{0}+L^{3} \psi_{4}^{0}\right)\right|_{l=1} \cong 3\left(L \psi_{2}^{0}\right)\right|_{l=1} \ldots
$$

It is this relationship that had, in the first model, its counterpart in the arbitrary assumption of Eq.(49). Now it has a justification and a geometric meaning. Also it does not contain an arbitrary factor $\alpha$. The quantity $\left.\psi_{1}^{0}\right|_{l=1}$ is uniquely defined by the choice of $L(u, \zeta, \bar{\zeta})$.

Our second and third models depend on two different, but related, choices of $L(u, \zeta, \bar{\zeta})$. For the third model, we choose the $L(u, \zeta, \bar{\zeta})$ to determine an arbitrary asymptotically shear-free null geodesic congruence, via Eqs.(22) and (25), while for the second model we choose the $L(u, \zeta, \bar{\zeta})$ associated with flat-space shearfree null geodesic congruences, Eqs.(33) and (32). The $L(u, \zeta, \bar{\zeta})$ in either case 
is determined by an arbitrary complex world-line $\xi^{a}(\tau)$, which contains the information about the center of mass world-line and the spin. Though the third model is more consistent and has a more logical basis, for two reasons we first discuss model two. It is easier to work with and the final results are very similar to those of the third model.

Using the flat-space $L(u, \zeta, \bar{\zeta})$, working with terms up to $2^{\text {nd }}$ order, with frequent use of Clebsch-Gordon expansions, we find, from Eq.(62), that

$$
\psi_{1 i}^{0}=3 \Upsilon\left[\xi^{i}(w)+i \frac{1}{2} \epsilon_{k j i} v^{k} \xi^{j}\right]+i \frac{3 \sqrt{2}}{2} \epsilon_{l j i} \psi_{2 j}^{0} \xi^{l}-\frac{18}{5} \psi_{2 i j}^{0} \xi^{j}
$$

There are two things to immediately notice: 1 . since $\Upsilon$ is proportional to the mass, the first term has exactly the same form as in the first model with $\xi^{i}$ now replacing the $\lambda^{i}$ as the 'complex center of mass' and 2 . the $\psi_{1 i}^{0}$ is fully determined without the constant $\alpha$. In addition, new terms have appeared, some of which will have immediate physical meaning. From the Bianchi Identity, Eq. (10), using Eq. (63) and the reality conditions, we have for the $l=0$ component of $\psi_{2}^{0}$,

$$
\begin{aligned}
& M=M_{0}-\frac{288 c}{5 G}\left(\xi_{R}^{i j} \xi_{R}^{i j \prime}+\xi_{I}^{i j} \xi_{I}^{i j \prime}\right) \\
& M_{0}=-\frac{c^{2}}{2 \sqrt{2} G} \Upsilon_{R}(w) \\
& \Upsilon_{I}=\frac{24(24) \sqrt{2}}{5 c}\left(\xi_{R}^{i j} \xi_{I}^{i j \prime}-\xi_{I}^{i j} \xi_{R}^{i j \prime}\right)
\end{aligned}
$$

which are identical to those of the first model. However for the $l=1$ components new terms appear:

For the momentum $\vec{P}$ we have

$$
\begin{aligned}
P^{k} & =M_{0} v_{R}^{k}+\frac{M_{0}}{c}\left(v_{R}^{i} v_{I}^{j}-\xi_{I}^{i} v_{R}^{j \prime}-\left(\xi_{R}^{i} v_{I}^{j}\right)^{\prime}\right) \epsilon_{i j k}+R^{k} \\
R^{k} & =-\frac{3(24) c^{2}}{5 G \sqrt{2}}\left(\xi_{R}^{i} \xi_{R}^{i k}+\xi_{I}^{i} \xi_{I}^{i k}\right)^{\prime}-\frac{(24)^{2} c^{2}}{5 G}\left(\xi_{I}^{i l} \xi_{R}^{i j \prime}-\xi_{R}^{i l} \xi_{I}^{i j \prime}\right) \epsilon_{l j k} .
\end{aligned}
$$

The $l=1$ part of reality condition yields an expression that consists of two types of terms, total time derivatives and the others. We define it as the conservation of angular momentum equation:

$$
\begin{aligned}
J^{k \prime} & =-\frac{(24)^{2} c^{3}}{5 G}\left(\xi_{R}^{i l} \xi_{R}^{i j \prime}+\xi_{I}^{i l} \xi_{I}^{i j \prime}\right) \epsilon_{l j k}, \\
J^{k} & =M_{0} c \xi_{I}^{k}+M_{0}\left(\xi_{R}^{i} v_{R}^{j}-\xi_{I}^{i} v_{I}^{j}\right) \epsilon_{i j k}-\frac{3(24) c^{3}}{5 \sqrt{2} G}\left(\xi_{I}^{i} \xi_{R}^{i k}-\xi_{R}^{i} \xi_{I}^{i k}\right) .
\end{aligned}
$$

The angular momentum flux is the same as in the first model. However the angular momentum itself has much more physical content. The first term 


$$
S^{k}=M_{0} c \xi_{I}^{k}
$$

is again identified as the intrinsic spin, while the second term is precisely the orbital angular momentum, $\mathbf{r} \times \mathbf{p}$, while the third term is the contribution from spin-precession. The fourth term is new, involving dipole and quadrupole coupling. This could be considered as a prediction of the theory though it probably is untestable. With these identifications, going back to $P^{k}$ in Eq.(64), we observe that the second term

$$
\frac{M}{c} \xi_{I}^{i \prime} v_{R}^{j} \epsilon_{i j k}=c^{-2} S^{i \prime} v_{R}^{j} \epsilon_{i j k}=c^{-2}\left(\mathbf{S}^{\prime} x \mathbf{v}\right)_{k}
$$

is the Mathisson-Papapetrou contribution to the linear momentum.

Remark 4. Many of the kinematic expressions for the physical quantities have been given up to second order. When the dynamic equations are used, it often turns out that these quantities are really of higher order and should be neglected for second order considerations. We felt that for purposes of understanding their kinematic role, their inclusion here was important.

The equations of motion for the position vector, $\xi_{R}^{k}$, are obtained by substituting the $\vec{P}$ from Eq.(64) into the momentum loss equation

$$
P^{k \prime}=F^{k} \equiv \frac{192 c^{2}}{5 G}\left(v_{I}^{l j} v_{R}^{i j}-v_{R}^{l j} v_{I}^{i j}\right) \epsilon_{i l k}
$$

yielding

$$
M_{0} v_{R}^{k \prime}-\frac{M_{0}}{c}\left(\xi_{R}^{i} v_{I}^{j \prime}+\xi_{I}^{i} v_{R}^{j \prime}\right)^{\prime} \epsilon_{i j k}+R^{k \prime}=\frac{384 c^{2}}{5 G} v_{I}^{l j} v_{R}^{i j} \epsilon_{i l k} .
$$

This again is Newton's $2^{\text {nd }}$ law but with spin-coupling forces, radiation reaction and momentum recoil.

Though this second model is far superior to the first model it still has certain deficiencies. It does not yield the correct dynamics for the algebraically special metrics. In other words the dynamical equations would differ from those of the algebraically special metrics. Also there is a lack of logical consistency in this treatment. We have used the $L(u, \zeta, \bar{\zeta})$ for the flat-space shear-free null geodesic congruence. We should have used the $L(u, \zeta, \bar{\zeta})$ associated with the asymptotically shear-free congruence, i.e., the $L(u, \zeta, \bar{\zeta})$ from Eq.(22). We would then have the null rotated Weyl component, $\psi_{1 i}^{* 0}$, based on an asymptotically shear-free null congruence so that the quadratic terms in the shear, that would normally appear in the definition of angular momentum, would be now absent, [See Remark 3.] and our procedure of setting $\psi_{1 i}^{* 0}=0$ would be consistent with all definitions of center of mass and angular momentum at the complex 'origin'.

\subsection{The Third Identification Method or Physical Model}

The third method is, as we mentioned earlier, basically the same as the second method. So rather than repeat ourselves with the vacuum 'asymptotically shear- 
free', $L(u, \zeta, \bar{\zeta})$, we will give the results for the asymptotically-flat EinsteinMaxwell equations. Since the method and arguments are so similar to those of method two and the detailed calculations are so long, we will simply summarize the results.

The physical identifications are substantially changed by the presence of the Maxwell field. For the vacuum case our results apply to the general asymptotically flat situation. For asymptotically flat GR, with the Maxwell field, the situation is different. It turns out that the Weyl tensor condition (62) has a counterpart for the Maxwell tensor. In other words, the $l=1$ part of a null rotated $\phi_{0}^{0}$, i.e., $\phi_{0 i}^{0 *}$ when set to zero, determines a different complex world-line (the complex center of charge) for which the electric and magnetic dipole moments vanish. We consider only the special case where the gravitational and electromagnetic world-lines coincide. In some sense this coincidence of worldlines implies that the source has a restricted structure and is relatively 'simple'. We take this as the meaning of a gravitational elementary particle.

We start with a very general cut function and its inverse that came from a given Bondi shear, i.e., from Eqs.(25) and (22):

$$
\begin{aligned}
u & \equiv \frac{w}{\sqrt{2}}=X(\tau, \zeta, \bar{\zeta})=\frac{1}{\sqrt{2}} \xi^{0}(\tau)-\frac{1}{2} \xi^{i}(\tau) Y_{1 i}^{0}(\zeta, \bar{\zeta})+\xi^{i j}(\tau) Y_{2 i j}^{0}(\zeta, \bar{\zeta})+\ldots \\
\tau & =T(u, \zeta, \bar{\zeta})=w+\frac{\sqrt{2}}{2} \xi^{i}(w) Y_{1 i}^{0}(\zeta, \bar{\zeta})-\sqrt{2} \xi^{i j}(w) Y_{1 i j}^{0}(\zeta, \bar{\zeta})+\ldots \\
L & =\xi^{i}(\tau) Y_{1 i}^{1}(\zeta, \bar{\zeta})-6 \xi^{i j}(\tau) Y_{2 i j}^{1}(\zeta, \bar{\zeta}) \\
\xi^{a} & =\left(\xi^{0}, \xi^{i}(\tau)=\left(\tau, \xi^{i}(\tau)=\xi_{R}^{a}(w)+i \xi_{I}^{a}(w)\right.\right. \\
\xi^{a \prime} & \equiv v^{a}(w)=v_{R}^{a}(w)+i v_{I}^{a}(w)
\end{aligned}
$$

After eliminating $\tau$, Eq.(62) yields the expression for $\psi_{1 i}^{0}$ :

$$
\begin{aligned}
\psi_{1 i}^{0} & =3 \Upsilon\left[\xi^{i}(w)+i \frac{1}{2} \epsilon_{k j i} v^{k} \xi^{j}+N^{i}\right]+i \frac{3 \sqrt{2}}{2} \epsilon_{l j i} \psi_{2 j}^{0} \xi^{l}-\frac{18}{5} \psi_{2 i j}^{0} \xi^{j} \\
& -i \frac{6 \cdot 36 \sqrt{2}}{5} \psi_{2 k j}^{0} \xi^{k l} \epsilon_{l j i}-\frac{6 \cdot 18}{5} \psi_{2 j}^{0} \xi^{i j} \\
N^{i} & =\frac{6 \sqrt{2}}{5} v^{k} \xi^{k i}-\frac{18 \sqrt{2}}{5} v^{k i} \xi^{k}+i \frac{144}{5} \epsilon_{j m i} v^{k j} \xi^{m k}
\end{aligned}
$$

Aside: Later, for the vacuum case, we will associate the imaginary part of Eq.(74) as the total angular momentum.

Integrating the Maxwell equations leads to 


$$
\begin{aligned}
\phi_{1 i}^{0} & =\sqrt{2} Q\left[v^{i}(w)+i \frac{1}{2} \epsilon_{i j l} v^{i \prime} \xi^{j}+N^{i \prime}\right]+i \sqrt{2} Q \epsilon_{k j i} v^{j \prime} \xi^{k}-\frac{2}{5}\left(\phi_{0 k i}^{0 \prime} \xi^{k}\right)^{\prime}-\frac{72}{5} Q v^{j} v^{i j} \\
& +i \frac{24 \sqrt{2}}{15} \epsilon_{l j i} \phi_{0 k j}^{0 \prime \prime} \xi^{l k}-i \frac{24 \sqrt{2}}{5} \epsilon_{j l i}\left(\phi_{0 m l}^{0 \prime} \xi^{j m}\right)^{\prime} \\
\phi_{2 i}^{0} & =-2 Q\left[v^{i \prime}+i \frac{1}{2} \epsilon_{i j l}\left(v^{i \prime} \xi^{j}\right)^{\prime}+N^{i \prime \prime}\right]-i 2 Q \epsilon_{k j i}\left(v^{j \prime} \xi^{k}\right)^{\prime}+\frac{2 \sqrt{2}}{5}\left(\phi_{0 k i}^{0 \prime} \xi^{k}\right)^{\prime \prime} \\
& +\frac{72 \sqrt{2}}{5} Q\left(v^{j} v^{i j}\right)^{\prime}-\frac{48}{15} i \epsilon_{l j i}\left(\phi_{0 k j}^{0 \prime \prime} \xi^{l k}\right)^{\prime}+i \frac{48}{5} \epsilon_{j l i}\left(\phi_{0 m l}^{0 \prime} \xi^{j m}\right)^{\prime \prime}
\end{aligned}
$$

From the Bianchi Identity Eq.(15) we obtain

$$
\begin{aligned}
P^{k} & =M v_{R}^{k}+\frac{M_{0}}{c}\left(v_{R}^{i} v_{I}^{j}-\xi_{I}^{i} v_{R}^{j \prime}-\left(\xi_{R}^{i} v_{I}^{j}\right)^{\prime}\right) \epsilon_{i j k}-\frac{2 Q^{2}}{3 c^{3}} v_{R}^{k \prime} \\
& +\frac{2 Q^{2}}{3 c^{4}}\left[2 \xi_{I}^{i} v_{R}^{j \prime}-\xi_{R}^{i} v_{I}^{j \prime}+v_{R}^{i} v_{I}^{j}\right]^{\prime} \epsilon_{i j k}+\Pi^{k} \\
\Pi^{k} & =-\frac{M}{c}\left(\frac{6 \sqrt{2}}{5}\left[8\left(\xi_{R}^{k i} v_{R}^{i}-\xi_{I}^{k i} v_{I}^{i}\right)+3\left(v_{R}^{k i} \xi_{R}^{i}-v_{I}^{k i} \xi_{I}^{i}\right)\right]^{\prime}+\frac{144}{5}\left(v_{R}^{i l \prime} \xi_{I}^{i j}+v_{I}^{i l \prime} \xi_{R}^{i j}\right) \epsilon_{l j k}\right) \\
& +\frac{Q^{2}}{3 c^{4}}\left(\frac{18(6) \sqrt{2}}{5}\left(v_{R}^{i \prime} \xi_{R}^{i k}+v_{I}^{i \prime} \xi_{I}^{i k}\right)^{\prime}+\frac{96 \sqrt{2}}{5}\left(v_{R}^{k i} v_{R}^{i}-v_{I}^{k i} v_{I}^{i}\right)^{\prime}\right. \\
& \left.-\frac{12 \sqrt{2}}{5}\left(\xi_{R}^{k i} v_{R}^{i \prime}-\xi_{I}^{k i} v_{I}^{i \prime}\right)^{\prime}+\frac{(36) \sqrt{2}}{5}\left(v_{R}^{k i \prime} \xi_{R}^{i}-v_{I}^{k i \prime} \xi_{I}^{i}\right)^{\prime}+\frac{288}{5}\left(v_{R}^{i l \prime} \xi_{I}^{i j}+v_{I}^{i l \prime} \xi_{R}^{i j}\right)^{\prime} \epsilon_{l j k}\right) \\
& +\frac{Q}{3 c^{4}}\left(\frac{24}{5 \sqrt{2} c}\left(D_{E}^{i j \prime \prime} \xi_{I}^{i l}+D_{M}^{i j \prime \prime} \xi_{R}^{i l}\right)^{\prime \prime} \epsilon_{l j k}-\frac{1}{5 c}\left(\xi_{R}^{i} D_{E}^{i k \prime \prime}-\xi_{I}^{i} D_{M}^{i k \prime \prime}\right)^{\prime \prime}\right. \\
& \left.-\frac{24}{15 \sqrt{2} c}\left(D_{E}^{i j \prime \prime \prime} \xi_{I}^{i l}+D_{M}^{i j \prime \prime \prime} \xi_{R}^{i l}\right)^{\prime} \epsilon_{l j k}-\frac{1}{5 c}\left(v_{R}^{i \prime} D_{E}^{i k \prime \prime}+v_{I}^{i \prime} D_{M}^{i k \prime \prime}\right)+\frac{1}{5 c}\left(v_{R}^{i} D_{E}^{i k \prime \prime \prime}+v_{I}^{i} D_{M}^{i k \prime \prime \prime}\right)\right) \\
& +\frac{4}{45 c^{4}}\left(D_{M}^{i j \prime \prime} D_{E}^{i l \prime \prime \prime}-D_{E}^{i j \prime \prime} D_{M}^{i l \prime \prime \prime}\right) \epsilon_{l j k}-\frac{36 \sqrt{2} c^{2}}{5 G}\left(\xi_{R}^{i} \xi_{R}^{i k}+\xi_{I}^{i} \xi_{I}^{i k}\right)^{\prime} \\
& +\frac{2(24)^{2} c^{2}}{5 G}\left(v_{R}^{i j} \xi_{I}^{i l}+\xi_{R}^{i j} v_{I}^{i l}\right) \epsilon_{l j k} .
\end{aligned}
$$

$D_{E}^{l j}$ and $D_{M}^{i j}$ are respectively the electric quadrupole and magnetic quadrupole moments found from the $l=2$ radiation term in the solution of the Maxwell equations. All non-linear terms involving the quadrupole terms are gathered into the $\Pi^{k}$. tions

The vanishing of the imaginary part of the reality condition yields the rela-

$J^{k \prime}=\frac{2 Q^{2}}{3 c^{3}}\left(v_{R}^{i \prime} v_{R}^{j}+v_{I}^{i \prime} v_{I}^{j}\right) \epsilon_{l j k}+\frac{1}{90 c^{5}}\left(D_{E}^{i j \prime \prime} D_{E}^{i l \prime \prime \prime}+D_{M}^{i j \prime \prime} D_{M}^{i l \prime \prime \prime}\right) \epsilon_{l j k}-\frac{(24)^{2} c^{3}}{5 G}\left(\xi_{R}^{i l} v_{R}^{i j}+\xi_{I}^{i l} v_{I}^{i j}\right) \epsilon_{l j k}$ 
where $J^{k}$, identified (from the dynamics rather than through the conventional symmetry argument) as the total angular momentum, is given by

$$
\begin{aligned}
J^{k} & \equiv M c \xi_{I}^{k}+M\left(\xi_{R}^{i} v_{R}^{j}-\xi_{I}^{i} v_{I}^{j}\right) \epsilon_{i j k}+\frac{2 Q^{2}}{3 c^{2}} v_{I}^{k}-\frac{2 Q^{2}}{3 c^{3}}\left(\xi_{R}^{i} v_{R}^{j \prime}+2 \xi_{I}^{i} v_{I}^{j \prime}\right) \epsilon_{i j k}+K^{k} \\
K^{k} & =-M\left(\frac{6 \sqrt{2}}{5}\left[8\left(\xi_{R}^{k i} v_{I}^{i}+\xi_{I}^{k i} v_{R}^{i}\right)+3\left(v_{R}^{k i} \xi_{I}^{i}+v_{I}^{k i} \xi_{R}^{i}\right)\right]-\frac{144}{5}\left(v_{R}^{i l} \xi_{R}^{i j}-v_{I}^{i l} \xi_{I}^{i j}\right) \epsilon_{l j k}\right) \\
& -\frac{Q^{2}}{3 c^{3}}\left(-\frac{18(6) \sqrt{2}}{5}\left(v_{R}^{i \prime} \xi_{I}^{i k}-v_{I}^{i \prime} \xi_{R}^{i k}\right)+\frac{96 \sqrt{2}}{5}\left(v_{R}^{k i} v_{I}^{i}+v_{I}^{k i} v_{R}^{i}\right)\right. \\
& \left.-\frac{12 \sqrt{2}}{5}\left(\xi_{R}^{k i} v_{I}^{i \prime}+\xi_{I}^{k i} v_{R}^{i \prime}\right)+\frac{(36) \sqrt{2}}{5}\left(v_{R}^{k i \prime} \xi_{I}^{i}+v_{I}^{k i \prime} \xi_{R}^{i}\right)-\frac{288}{5}\left(v_{R}^{i l \prime} \xi_{R}^{i j}-v_{I}^{i l \prime} \xi_{I}^{i j}\right) \epsilon_{l j k}\right) \\
& +\frac{Q}{15 c^{4}}\left(\xi_{R}^{i} D_{M}^{i k \prime \prime \prime}+\xi_{I}^{i} D_{E}^{i k \prime \prime \prime}+2 v_{I}^{i} D_{E}^{i k \prime \prime}+4 \sqrt{2}\left(2 \xi_{R}^{i l} D_{E}^{i j \prime \prime \prime}-2 \xi_{I}^{i l} D_{M}^{i j \prime \prime \prime}\right.\right. \\
& \left.\left.+3 v_{R}^{i l} D_{E}^{i j \prime \prime}-3 v_{I}^{i l} D_{M}^{i j \prime \prime}\right) \epsilon_{l j k}\right)-\frac{36 \sqrt{2} c^{3}}{5 G}\left(\xi_{I}^{i} \xi_{R}^{i k}-\xi_{R}^{i} \xi_{I}^{i k}\right) .
\end{aligned}
$$

The justification for calling this the angular momentum is the same as in the previous model, coming from the sum of terms interpretable as the spin, orbital and precessional moments.

In the absence of a Maxwell field $J^{k}$ is simply proportional to the imaginary part of $\psi_{1 i}^{0}$,

$$
J^{i}=-\left.\frac{\sqrt{2} c^{3}}{12 G} \psi_{1 i}^{0}\right|_{I} .
$$

Remark 5. In the published literature [4] there are ambiguities in the definition of angular momentum. For us, because of our approximations, these ambiguities disappear, i.e., the ambiguous terms are higher order. See Appendix 7.4 .

Our last results arise from are the mass/energy loss equation and the momentum loss equation. The later being the dynamic equations for $\xi_{R}^{i}$. By substituting the $P^{k}$ of Eq.(177) into the Bianchi identity, Eq.(16) we find

$$
\begin{aligned}
M^{\prime} & =-\frac{G}{5 c^{7}}\left(Q_{\text {Mass }}^{i j \prime \prime \prime} Q_{\text {Mass }}^{i j \prime \prime \prime}+Q_{\text {Spin }}^{i j \prime \prime \prime} Q_{\text {Spin }}^{i j \prime \prime}\right)-\frac{2 Q^{2}}{3 c^{5}}\left(v_{R}^{i \prime} v_{R}^{i \prime}+v_{I}^{i \prime} v_{I}^{i \prime}\right) \\
& -\frac{1}{180 c^{7}}\left(D_{E}^{i j \prime \prime \prime} D_{E}^{i j \prime \prime \prime}+D_{M}^{i j \prime \prime \prime} D_{M}^{i j \prime \prime \prime}\right) \\
P^{k \prime} & =F^{k} \equiv \frac{2 G}{15 c^{6}}\left(Q_{S p i n}^{l j \prime \prime \prime} Q_{M a s s}^{i j \prime \prime \prime}-Q_{M a s s}^{l j \prime \prime \prime} Q_{S p i n}^{i j \prime \prime \prime}\right) \epsilon_{i l k}-\frac{Q^{2}}{3 c^{4}}\left(v_{I}^{l \prime} v_{R}^{i \prime}-v_{R}^{l \prime} v_{I}^{i \prime}\right) \epsilon_{i l k} \\
& +\frac{Q}{15 c^{5}}\left(v_{R}^{j \prime} D_{E}^{j k \prime \prime \prime}+v_{I}^{j \prime} D_{M}^{j k \prime \prime \prime}\right)+\frac{1}{540 c^{6}}\left(D_{E}^{l j \prime \prime \prime} D_{M}^{i j \prime \prime \prime}-D_{M}^{l j \prime \prime \prime} D_{E}^{i j \prime \prime \prime}\right) \epsilon_{i l k}
\end{aligned}
$$

where we have now replaced the $\xi^{i j}$ by the more physical variables, the quadrupoles, $\left(Q_{\text {Mass }}^{i j \prime \prime}, Q_{S p i n}^{i j \prime \prime}\right)$ via

$$
\xi^{i j}=\left(\xi_{R}^{i j}+i \xi_{I}^{i j}\right)=\frac{G}{12 \sqrt{2} c^{4}}\left(Q_{\text {Mass }}^{i j \prime \prime}+i Q_{S p i n}^{i j \prime \prime}\right) .
$$


Writing out the equations of motion in detail is long and not completely enlightening, There are many non-linear terms whose meanings, other than they are interpretable as gravitational radiation reaction, are not clear. Instead we will write out a truncated version of Eq. (83), hiding these terms in a single symbol, $R^{k}$, so that we have

$$
M v_{R}^{k \prime}+v_{R}^{k} M^{\prime}-\frac{2 Q^{2}}{3 c^{3}} v_{R}^{k \prime \prime}+R^{k}=F^{k} .
$$

There are several things to notice here. First of all strictly speaking, we should ignore the term $v_{R}^{k} M^{\prime}$ since it is third order because $M^{\prime}$ already is second order from Eq. (82). We keep it, understanding its suspect nature, because it is potentially so important. For the moment ignore $R^{k}$ and $F^{k}$ and consider only the second term in the mass loss, i.e., the electric dipole energy loss, so that Eq. (85) becomes

$$
M v_{R}^{k \prime}-v_{R}^{k} \frac{2 Q^{2}}{3 c^{5}} v_{R}^{i \prime} v_{R}^{i \prime}-\frac{2 Q^{2}}{3 c^{3}} v_{R}^{k \prime \prime}=0
$$

These 24, 25] are the classical equations of motion for a charged particle that contain the well known electromagnetic radiation reaction force and exhibit the unstable run-away behavior. (The cubic term, though not sufficient large to stabilize the equation, has the correct sign for stabilization.) This classical equation has been obtained without the usual model building or mass renormalization. Returning to Eq. (85), we see that in addition to the $R^{k}$ and $F^{k}$, there are the extra terms in $M^{\prime}$ coming from gravitational and electromagnetic quadrupole radiation. It is very hard to see the consequences these terms though one can see that they are on the side of stabilization. It is then easy to conjecture that coupling electrodynamics with general relativity stabilizes the equations of motion.

There are other comments concerning our results that should be made.

- The mass/energy loss equation contains both the gravitational radiation quadrupole expression (this has been adjusted by definition) and the classical electromagnetic dipole and quadrupole radiation expressions that come straight from the construction. This allows us to identify

$$
\mu^{k}=Q \xi_{I}^{k}
$$

as the magnetic dipole moment, so that with the spin definition, $S^{k}=M c \xi_{I}^{k}$, we obtain the Dirac value of the gyromagnetic ratio, i.e.,

$$
g=2 .
$$

- The angular momentum expression, Eq.(80), contains the spin, the orbital angular momentum and a precession contribution. There is now a prediction that there is a charge-spin contribution to the total $J$, i.e., $\frac{2 Q^{2}}{3 M c^{3}} S^{k \prime}$, as well has higher order corrections.

- Probably the strongest argument for the validity of this approach to extracting physical information from the asymptotic field is the observation that 
in the angular momentum loss equation, Eq.(79), the dipole contribution to the angular momentum flux coming just from the electromagnetic field, i.e.,

$$
\frac{2 Q^{2}}{3 c^{3}}\left(v_{R}^{i \prime} v_{R}^{j}+v_{I}^{i \prime} v_{I}^{j}\right) \epsilon_{l j k}
$$

exactly coincides with the classical electrodynamic angular momentum flux [25]. This result was obtained with no a priori expectations.

\section{BMS Invariance}

Our results concerning the identifications of physical quantities from the asymptotic fields were all obtained in an arbitrary but specific Bondi coordinate/tetrad system. The question is what are the relations between the same quantities but calculated in a different Bondi coordinate/tetrad system? In other words, we want to know the transformation properties of our physical variables under the action of the BMS group [8, 9, 15, 16, 14]. As we pointed out in Sec. II, the BMS group is composed of two parts, the supertranslations and the Lorentz transformations given respectively by

$$
\begin{aligned}
\widehat{u} & =u+\alpha(\zeta, \bar{\zeta}) \\
(\widehat{\zeta}, \bar{\zeta}) & =(\zeta, \bar{\zeta})
\end{aligned}
$$

with $\alpha(\zeta, \bar{\zeta})$ an arbitrary smooth function on the sphere considered now to be small i.e., as a first order quantity and

$$
\begin{aligned}
\widehat{u} & =K u \\
K & =\frac{1+\zeta \bar{\zeta}}{(a \zeta+b)(\bar{a} \bar{\zeta}+\bar{b})+(c \zeta+d)(\bar{c} \bar{\zeta}+\bar{d})} \\
\widehat{\zeta} & =\frac{a \zeta+b}{c \zeta+d} ; \quad a d-b c=1 .
\end{aligned}
$$

with $(a, b, c, d)$ the complex parameters of $\mathrm{SL}(2, \mathrm{C})$. The invariance under supertranslations is actually subtle and requires a bit of thought while the invariance under the Lorentz group, though straightforward, requires some more technical background. Since all our calculations were done under the assumption of second order perturbations off a Reissner-Nordstrom background, we must keep the BMS transformations small, i.e., close to the identity.

\subsection{The Supertranslations}

We first treat supertranslation invariance.

Starting on $\mathfrak{I}^{+}$with some arbitrary but given Bondi coordinates $(u, \zeta, \bar{\zeta})$ and 
tetrad $(l, n, m, \bar{m})$ we saw that there was a null rotation, Eq.(77),

$$
\begin{aligned}
l^{*} & =l+L \bar{m}+\bar{L} m+L \bar{L} n \\
m^{*} & =m+L n \\
n^{*} & =n
\end{aligned}
$$

that was determined by the choice of the angle field $L(u, \zeta, \bar{\zeta})$. The $L(u, \zeta, \bar{\zeta})$ was then determined first by the asymptotic shear-free condition, leaving the freedom in the $l=(0,1)$ harmonic coefficients, and then by the requirement that the $l=1$ harmonic component of the 'rotated' Weyl component, $\psi_{1}^{0 *}$, i.e., $\left.\psi_{1}^{0 *}\right|_{l=1}=0$, should vanish. The important point to note is that the Weyl tensor component $\psi_{1}^{0 *}$ is determined by the new tetrad, $\left(l^{*}, n^{*}, m^{*}, \bar{m}^{*}\right)$, It is a geometric structure given independent of the choice of coordinates - depending on the fixed known $\left(^{*}\right)$-tetrad. If we have a second Bondi system with Bondi tetrad $(\widehat{l}, \widehat{n}, \widehat{m}, \overline{\widehat{m}})$, it will have been obtained by a different null rotation via some other angle field, $\widehat{L}$, from the $(l, n, m, \bar{m})$ tetrad. This simply means that there is a different null rotation, going now from the ( )-tetrad to the previously determined $(*)$-tetrad. The $\left.\psi_{1}^{0 *}\right|_{l=1}$ thus remains zero when this harmonic is extracted from the $\psi_{1}^{0 *}$ when the ' $u$ ' is held constant. There however is a serious issue that must be raised. When we extracted the $l=1$ part of $\psi_{1}^{0 *}$, it was done at constant value of the Bondi ' $u$ '. But now, after the supertranslation, the harmonic decomposition should be done at constant ' $\widehat{u}$ '. However, it turns out, because of the first order BMS supertranslation, $\widehat{u}=u+\alpha(\zeta, \bar{\zeta})$, the $\left.\psi_{1}^{0 *}\right|_{l=1}$ part of $\psi_{1}^{0 *}$, now obtained by holding ' $\widehat{u}$ ' constant, remains zero up to second order. So up to our accuracy, our results are supertranslation invariant. The more difficult issue of how to deal with finite supertranslations will be discussed in the conclusion.

\subsection{Lorentz Transformations}

To deal with the Lorentz subgroup of the BMS group requires a review of the representation theory.

The theory of the representations of the Lorentz group was beautifully described by Gelfand, Graev and Vilenkin[13] using homogeneous functions of two complex variables as the representation space. We will summarize these ideas using an equivalent method 14 namely by using spin-weighted functions of the sphere as the representation spaces. Representations are labeled by either of two numbers $\left(n_{1}, n_{2}\right)$ or $(s, w)$, with $\left(n_{1}, n_{2}\right)=(w-s+1, w+s+1)$. The ' $s$ ' is referred to as the spin weight and ' $w$ ' as the conformal weight. The representations are referred to as $D_{\left(n_{1}, n_{2}\right)}$. The special case of irreducible unitary representations, which occur when $\left(n_{1}, n_{2}\right)$ are not integers, are not of interest to us and will not be discussed. We will consider only the case when $\left(n_{1}, n_{2}\right)$ are integers so that the $(s, w)$ then can take on integer or half integer values. The representation space, for each $(s, w)$, are the functions on the sphere, $\eta_{(s, w)}(\zeta, \bar{\zeta})$, that can be 
expanded in spin-weighted spherical harmonics, ${ }_{s} Y_{l m}(\zeta, \bar{\zeta})$, so that

$$
\eta_{(s, w)}(\zeta, \bar{\zeta})=\sum_{l=s}^{\infty} \eta_{(l m)}{ }_{s} Y_{l m}(\zeta, \bar{\zeta})
$$

and transform under the Lorentz group, Eq.910, as

$$
\widehat{\eta}_{(s, w)}(\widehat{\zeta}, \overline{\widehat{\zeta}})=e^{i s \lambda} K^{w} \eta_{(s, w)}(\zeta, \bar{\zeta})
$$

with

$$
\begin{aligned}
e^{i \lambda} & =\frac{c \zeta+d}{\bar{c} \bar{\zeta}+\bar{d}}, \\
K & =(1+\zeta \bar{\zeta})[(a \zeta+b)(\bar{a} \bar{\zeta}+\bar{b})+(c \zeta+d)(\bar{c} \bar{\zeta}+\bar{d})]^{-1},
\end{aligned}
$$

These representations, in general, are neither irreducible nor totally reducible. For us the important point is that many of these representations do possess an invariant finite-dimensional subspace which (often) corresponds to the usual finite dimensional tensor representation space. Under the transformation. (94). the finite number of coefficients in these subspaces transform among themselves. It is this fact which we heavily utilize. More specifically we have two related situations: (1) when the $\left(n_{1}, n_{2}\right)$ are both positive integers, (or $\left.w>|s|\right)$, there will be a finite dimensional invariant subspace, $D_{\left(n_{1}, n_{2}\right)}^{+}$, and (2) when the $\left(-n_{1},-n_{2}\right)$ are both negative integers there will be an infinite dimensional invariant subspace, $D_{\left(-n_{1},-n_{2}\right)}^{-}$. One, however, can obtain a finite dimensional representation for each negative integer case by the following construction: One forms the factor space, $D_{\left(-n_{1},-n_{2}\right)} / D_{\left(-n_{1},-n_{2}\right)}^{-}$. This space is isomorphic to one of the finite dimensional spaces associated with the positive integers. The explicit form of the isomorphism, which is not needed here, is given in Held \& Newman 14.

Rather than give the full description of these invariant subspaces, which is available elsewhere, we will confine ourselves to the few cases of relevance to us.

I. For $s=0$ and $w=1,\left[\left(n_{1}, n_{2}\right)=(2,2)\right]$, the harmonics, $l=(0,1)$ form the invariant subspace. Applying this to the cut function, $X(\tau, \zeta, \bar{\zeta})$, we obtain from

$$
u=X_{(0,1)}=\frac{1}{\sqrt{2}} \xi^{0}(\tau)-\frac{1}{2} \xi^{i}(\tau) Y_{1 i}^{0}(\zeta, \bar{\zeta})+\xi^{i j}(\tau) Y_{2 i j}^{0}(\zeta, \bar{\zeta})+\ldots
$$

$$
\begin{aligned}
\text { Invar. subspace } & =\frac{1}{\sqrt{2}} \xi^{0}(\tau)-\frac{1}{2} \xi^{i}(\tau) Y_{1 i}^{0}(\zeta, \bar{\zeta}) \\
\xi^{a}(\tau) & =\left(\xi^{0}(\tau), \xi^{i}(\tau)\right)=\text { Lorentz vector }
\end{aligned}
$$

This allows us to single out, in a Lorentz invariant manner, the four $l=(0,1)$ harmonic coefficients of the cut-function $X(\tau, \zeta, \bar{\zeta})$ as a complex position vector.

II. The mass aspect,

$$
\Psi \equiv \Psi_{(0,-3)}=\Psi^{0}+\Psi^{i} Y_{1 i}^{0}+\Psi^{i j} Y_{2 i j}^{0}+\ldots
$$


is an $s=0$ and $w=-3,\left[\left(n_{1}, n_{2}\right)=(-2,-2)\right]$ quantity. The factor space is isomorphic to the finite dimensional positive integer space, $\left[\left(n_{1}, n_{2}\right)=(2,2)\right]$ and hence the harmonics coefficients of $l=(0,1)$ lie in the invariant subspace. From the isomorphism, (which does change the numerical coefficients) we can construct functions of the form Eq.(97), which, in turn, lead to

$$
P^{a}=\left(M c, P^{i}\right)=\text { Lorentz vector }
$$

This gives the justification for calling the $l=(0,1)$ harmonics of the mass aspect, a Lorentzian four-vector, $P^{a}$.

III. The Weyl tensor component, $\psi_{1}^{0}$, has $s=1$ and $w=-3$, $\left[\left(n_{1}, n_{2}\right)=\right.$ $(-3,-1)]$. The associated finite dimensional factor space is isomorphic to the finite part of the $s=-1, w=1,\left[\left(n_{1}, n_{2}\right)=(3,1)\right]$ representation. We have that

$$
\psi_{1}^{0} \equiv \psi_{1(1,-3)}^{0}=\psi_{1 i}^{0} Y_{1 i}^{1}+\psi_{1 i j}^{0} Y_{2 i j}^{1}+\ldots
$$

leads to the invariant subspace

$$
\text { Invariant subspace }=\psi_{1 i}^{0} Y_{1 i}^{-1} .
$$

The question of what finite tensor transformation does this correspond to is slightly more complicated than that of the previous examples of Lorentzian vectors. In fact it corresponds to the Lorentz transformations applied to (complex) self-dual antisymmetric two-index tensors. As an example from Maxwell theory, from a given $\mathbf{E}$ and $\mathbf{B}$, the Maxwell tensor, $F^{a b}$, and then its self-dual version

$$
W^{a b+}=F^{a b}+i F^{* a b}
$$

can be constructed. A Lorentz transformation applied to $W^{a b+}$ is equivalent 25], (see appendix B) to the same transformation applied to

$$
\psi_{1 i}^{0}=(\mathbf{E}+i \mathbf{B})_{i} .
$$

These observations allow us to assign invariant physical meaning to our identifications of the position vector, $\xi^{a}$, the Bondi momentum, $P^{a}$ and the angular momentum $J^{i}$.

\section{Discussion}

Starting from a very unorthodox point of view, we have tried to describe in the context of GR, (either in the vacuum case or for the Einstein-Maxwell equations), the equations of motion of an isolated charged, massive body (our gravitational elementary particle) that possesses both an intrinsic spin and quadrupole moments and can radiate both gravitational and electromagnetic radiation. The

point of view arises from considering a generalization of the algebraically special metrics where one can identify physical quantities and determine their evolution from the asymptotic field equations. 
For the algebraically special metrics, the shear-free null geodesic congruence that are associated with these metrics, automatically assigned kinematic variables (e.g., a position vector and an intrinsic spin) to the Bondi energymomentum four-vector. The Bondi evolution equations then became the equations of motion. Our generalization consisted in observing that the existence of a shear-free null geodesic congruence could be generalized to the existence of an asymptotically shear-free null geodesic congruence. Applying the same physical identifications as with the shear-free congruences leads in exactly the same manner to the more general equations of motion.

To test out if this result was accidental and, perhaps, other physical assignments would lead to similar equations of motion, we tried two alternate strategies. They both were found to be lacking. They were not as natural or as physically meaningful as the third identification method.

Since we are far from any of the standard or default approaches to the description of motion in GR it would be appropriate to summarize our results.

Looking at the asymptotic Einstein-Maxwell equations, the asymptotic shearfree conditions lead, in general, to two different complex world-lines in the space of complex Poincare translations acting on $\mathfrak{I}^{+}$, one from the Weyl tensor, the other from the Maxwell tensor. We have considered only the special case where the two world-lines coincide - defining this case as "elementary particles in GR".

Some results among others are:

- The mass has a kinematic correction term dependent on the variable quadrupole moment. This could perhaps be considered as a prediction.

- The Bondi linear three-momentum is expressed in kinematic variables, e.g., $M v^{k}, \frac{2 Q^{2}}{3 c^{3}} v_{R}^{k \prime}$ and the Mathisson-Papapetrou spin coupling, among others.

- The imaginary part of the complex position vector is identified with the specific intrinsic spin angular momentum. From the solutions to Maxwell's equations the magnetic moment is seen to be the charge, $q$, times the imaginary part of the position vector. This agrees with the algebraically special charged spinning metric and leads to the Dirac value of the gyromagnetic ratio, i.e., $g=2$. Though earlier we have defined a 'gravitational elementary particle' from this result, it should be noted that in the elementary particle community 29] it has been speculated that all charged elementary particles with spin have this property. One then has the question, what if any, is the relationship between the two types of particle?

- One of the strongest arguments for our interpretations comes from the $l=1$ part of the reality conditions, which is interpreted as the dynamics (the conservation law) for the total angular momentum. There is a total time derivative term of a quantity, $J^{k}$, that we define as the total angular momentum. It contains the spin, the orbital angular momentum and a precession term. The angular momentum flux contains three terms which come, respectively, from the gravitational quadrupole radiation, the electromagnetic quadrupole radiation and a term arising from the electromagnetic (electric and magnetic) dipole radiation. This latter term is identical to that calculated purely from electromagnetic theory 25$]$. 
- From the Bondi mass loss equation, we can identify from the flux terms, the gravitational quadrupole but also see that our identification of the electromagnetic dipole moments agrees with the predicted dipole energy loss.

- From the Bondi momentum loss we obtain the equations of motion. In a sense we "derive" Newton's $2^{\text {nd }}$ law , $F=M v$, where the force is a combination of electromagnetic radiation reaction 24, 25, gravitational radiation reaction and a "rocket" recoil force from the electromagnetic and gravitational momentum loss.

- Finally, we showed that each of the quantities that were identified as physical variables transformed appropriately under the Lorentz group, i.e., as Lorentzian tensorial objects.

- Our results follow from the existence of a well-defined geometric structure, namely the UCF, a unique one-complex parameter family of slices of null infinity. This suggests that the higher order coefficients in the harmonic expansion of the $\mathrm{UCF}$, e.g., $\xi^{i j}, \xi^{i j k}, \ldots$, should be identified with time-derivatives of the higher multipole moments.

There are other unfamiliar terms that could be thought of as predictions of this theoretical construct. How to possibly measure them is not at all clear.

- One interesting physical prediction concerns the contribution that the charge makes to the total angular momentum. Looking at the equation defining $J^{k}$, Eq. (80), we see the (linear) contribution from

$$
\frac{2 Q^{2}}{3 c^{2}} v_{I}^{k}=\frac{2 Q}{3 c^{2}} D_{M}^{k \prime},
$$

which is a coupling between the charge and the changing magnetic dipole moment.

The final item to be discussed concerns the invariance under the supertranslation subgroup of the BMS group.

An obvious question concerning the material described here concerns the issue of the extraction of the $l=(0,1)$ harmonics from different Weyl tensor components or from the universal cut function, $X$. We have consistently performed the extraction of the harmonic components on the cuts, $u=$ const., or on neighboring cuts, $\widehat{u}=$ const. $+\Delta$, that are close to the first set of cuts. To $2^{\text {nd }}$ order the results are unchanged. If we do go to arbitrary cuts, there is no reason for the extraction to lead to the same results. Our results thus appear to depend on the choice of cuts. In fact there is a canonical choice of cuts, i.e., a special one-parameter family of cuts, labeled by ' $s$ ', on which the extraction should always be performed and for which there is no ambiguity. In the text, the $u=$ const. cuts were sufficiently close to the canonical choice, so that, to $2^{\text {nd }}$ order, they were the same. In fact, in principle, we should have been doing all our calculations on the $s=$ const. cuts. It was, however, easier doing it with $u=$ const.

The question then is what are these canonical cuts? Returning to the complex universal cut-function

$$
u=X(\tau, \zeta, \bar{\zeta})
$$


we saw earlier that the $\tau$ had to be chosen so that the $u$ had real values. If we write

$$
\tau=s+i \Lambda(s, \zeta, \bar{\zeta})
$$

then one can show see appendix $\mathrm{A}\}$ that $\Lambda(s, \zeta, \bar{\zeta})$ can be chosen so that

$$
u=X(s+i \Lambda(s, \zeta, \bar{\zeta}), \zeta, \bar{\zeta})=\widehat{X}(s, \zeta, \bar{\zeta})
$$

is a real function of the real variable ' $s$ '. This is the construction of our canonical slicing.

\section{Acknowledgments}

G.S.O. acknowledges the financial support from CONACYT and Sistema Nacional de Investigadores (SNI-México). C.K. thanks CONICET and SECYTUNC for support.

\section{References}

[1] H. Weyl, Space-Time-Matter, Methuen, London , (1922).

[2] L. B. Szabados, "Quasi-Local Energy-Momentum and Angular Momentum in GR: A Review Article", in Living Reviews in Relativity, June 2005

[3] Adam D. Helfer, Gen. Relativity \& Gravity, 39, 2125-2147, (2007).

[4] Dray, T., and Streubel, M., Class. Quantum Grav., 1, 15-26, (1984).

[5] Ashtekar, A., and Winicour, J., J. Math. Phys., 23, 2410-2417, (1982).

[6] C. Kozameh, E.T. Newman and Gilberto Silva-Ortigoza, Class.Quantum.Grav., 24, 5479-5493, (2007).

[7] E.T. Newman, E. Couch, K. Chinnapared, A. Exton, A. Prakash, R. Torrence, J.Math.Phys.6, 918, (1965).

[8] H. Bondi, M.G.J. van der Burg and A.W.K. Metzner, Proc. R. Soc., London Ser. A 269, (1962) 21.

[9] R. Sachs, Proc. Roy. Soc. 270, 103, (1962).

[10] Ezra T. Newman and Roger Penrose, J.Math.Phys, 3 566-768, (1962).

[11] E. T. Newman and T. Unti, J. Math. Phys. 3, 891, (1962).

[12] E.T. Newman, K.P.Tod, in General Relativity and Gravitation, Vol 2, Alan Held, (ed.), Plenum (1980). 
[13] I.M.Gelfand, M.I.Graev and N.Ya.Vilenkin, in Generalized Functions, vol. 5: Integral geometry and problems of representation theory, Academic Press , New York, (1966).

[14] A. Held, E. T. Newman and R. Posadas, J. Math. Phys. 11, 3145 (1970).

[15] R. Sachs, Proc. Roy. Soc. 270, 103, (1962).

[16] R. Penrose, Phys. Rev. Ltt. 10, 66, (1963).

[17] C. Kozameh and E.T. Newman, Class. Quantum Grav. 22, 4659-4665, (2005).

[18] B. Aronson and E. T. Newman, J. Math. Phys. 13, 1847-51, (1972).

[19] R. Hansen, E.T. Newman, R. Penrose and K.P. Tod, Proc. Roy. Soc. A 363, 445, (1978).

[20] E.T. Newman and G. Silva-Ortigoza, Class.Quantum Grav., 23, 497-509, (2006).

[21] C. Kozameh, E.T. Newman and Gilberto Silva-Ortigoza, Class.Quantum.Grav.24, 1955-1979, (2007).

[22] E. T. Newman, Phys. Rev. D, 65, 104005, (2002).

[23] Ezra T. Newman, Class. Quantum Grav 21 3197-3221,(2004).

[24] W. Thirring, Principles of Quantum Electrodynamics, (p.25), Academic Press, NY and London, (1958).

[25] Landau and E. M. Lifschitz, Classical Theory of Fields, Addison-Wesley, Reading, Mass., (1962).

[26] I. Robinson and A. Trautman, Proc. Roy. Soc., London Ser., A 289, 463 (1962).

[27] C. J. Talbot, Commun. Math. Phys. 13, 45, (1969).

[28] C. Kozameh, E.T. Newman and G. Silva Ortigoza, Class. Quantum Grav. 23, 6599-6620, (2006).

[29] M. Rivas, J.M. Aguirregabiria, A. Hernandez, Phys. Letters.A, 257, 21-25, (1999). 


\section{Appendix}

\subsection{The Canonical Slicing}

To construct the canonical slicing we begin with the complex UCF

$$
\begin{aligned}
u & =X(\tau, \zeta, \bar{\zeta}) \\
& =\frac{1}{\sqrt{2}} \xi^{0}(\tau)-\frac{1}{2} \xi^{i}(\tau) Y_{1 i}^{0}(\zeta, \bar{\zeta})+\xi^{i j}(\tau) Y_{2 i j}^{0}(\zeta, \bar{\zeta})+\ldots
\end{aligned}
$$

and write

$$
\tau=s+i \lambda
$$

with $s$ and $\lambda$ real. The cut function can then be rewritten

$$
\begin{aligned}
u & =X(\tau, \zeta, \bar{\zeta})=X(s+i \lambda, \zeta, \bar{\zeta}) \\
& =\chi_{R}(s, \lambda, \zeta, \bar{\zeta})+i \chi_{I}(s, \lambda, \zeta, \bar{\zeta}),
\end{aligned}
$$

with real $\chi_{R}(s, \lambda, \zeta, \bar{\zeta})$ and $\chi_{I}(s, \lambda, \zeta, \bar{\zeta})$. The $\chi_{R}(s, \lambda, \zeta, \bar{\zeta})$ and $\chi_{I}(s, \lambda, \zeta, \bar{\zeta})$ are easily calculated from $X(\tau, \zeta, \bar{\zeta})$ by

$$
\begin{aligned}
\chi_{R}(s, \lambda, \zeta, \bar{\zeta}) & =\frac{1}{2}\{X(s+i \lambda, \zeta, \bar{\zeta})+\overline{X(s+i \lambda, \zeta, \bar{\zeta})}\} \\
\chi_{I}(s, \lambda, \zeta, \bar{\zeta}) & =\frac{1}{2}\{X(s+i \lambda, \zeta, \bar{\zeta})-\overline{X(s+i \lambda, \zeta, \bar{\zeta})}\}
\end{aligned}
$$

By setting

$$
\chi_{I}(s, \lambda, \zeta, \bar{\zeta})=0
$$

and solving for

$$
\lambda=\Lambda(s, \zeta, \bar{\zeta})
$$

we obtain the real slicing,

$$
u=\chi_{R}(s, \Lambda(s, \zeta, \bar{\zeta}), \zeta, \bar{\zeta})
$$

Remark 6. We remark without proof[6], that using the gauge freedom described early for the choice of the parameter $\tau$, we can normalize the real velocity vector, $v_{R}^{a}(s)=\xi_{R}^{a \prime}(s=\tau)$, to one, i.e., $\eta_{a b} v_{R}^{a}(s) v_{R}^{b}(s)=1$.

\subsection{Lorentzian Tensors}

In Sec. IV we pointed out that certain spin and conformal weighted function on the sphere carried finite dimensional representations of the Lorentz group, i.e., they carried information about Lorentzian tensor objects. As examples of this we will work out two specific cases. 
Starting with the Lorentz transformation

$$
\begin{aligned}
\widehat{\zeta} & =\frac{a \zeta+b}{c \zeta+d}, \quad a d-b c=1 \\
e^{i \lambda} & =\frac{c \zeta+d}{\bar{c} \bar{\zeta}+\bar{d}} \\
K & =\frac{(1+\zeta \bar{\zeta})}{[(a \zeta+b)(\bar{a} \bar{\zeta}+\bar{b})+(c \zeta+d)(\bar{c} \bar{\zeta}+\bar{d})]} \\
\widehat{\eta}_{(s, w)}(\widehat{\zeta}, \bar{\zeta}) & =e^{i s \lambda} K^{w} \eta_{(s, w)}(\zeta, \bar{\zeta})
\end{aligned}
$$

we choose the special transformation

$$
\begin{aligned}
\widehat{\zeta} & =a^{2} \zeta \\
e^{i \lambda} & =\frac{d}{\bar{d}}=\frac{\bar{a}}{a} \\
K & =\frac{a \bar{a}(1+\zeta \bar{\zeta})}{\left[(a \bar{a})^{2} \zeta \bar{\zeta}+1\right]}
\end{aligned}
$$

Example $1 \quad s=0$ and $w=1$

Applying this special transformation to the invariant subspace of an $s=0$ and $w=1$ quantity, e.g., to $u=X(\tau, \zeta, \bar{\zeta})$, we have

$$
\begin{aligned}
\widehat{\eta}_{(0,1)}(\widehat{\zeta}, \overline{\widehat{\zeta}}) & =K \eta_{(0,1)}(\zeta, \bar{\zeta}) \\
\widehat{\xi}^{a} l_{a}(\widehat{\zeta}, \bar{\zeta}) & =K \xi^{a} l_{a}(\zeta, \bar{\zeta}) \\
l_{a}(\zeta, \bar{\zeta}) & =\frac{\sqrt{2}}{2}\left(1, \frac{\zeta+\bar{\zeta}}{1+\zeta \bar{\zeta}},-i \frac{\zeta-\bar{\zeta}}{1+\zeta \bar{\zeta}}, \frac{-1+\zeta \bar{\zeta}}{1+\bar{\zeta}}\right)
\end{aligned}
$$

After using Eq.(115) in Eq.(117) and comparing the coefficients of $(1, \zeta, \bar{\zeta}, \zeta \bar{\zeta})$, we find that

$$
\begin{aligned}
\widehat{\xi}^{0} & =\frac{1}{2}\left(a \bar{a}+a^{-1} \bar{a}^{-1}\right) \xi^{0}+\frac{1}{2}\left(a^{-1} \bar{a}^{-1}-a \bar{a}\right) \xi^{3} \\
\widehat{\xi}^{3} & =\left(\frac{1}{2} a \bar{a}+\frac{1}{2} a^{-1} \bar{a}^{-1}\right) \xi^{3}+\left(\frac{1}{2} a^{-1} \bar{a}^{-1}-\frac{1}{2} a \bar{a}\right) \xi^{0} \\
\widehat{\xi}^{1}-i \widehat{\xi}^{2} & =\frac{\bar{a}}{a}\left(\xi^{1}-i \xi^{2}\right) \\
\widehat{\xi}^{1}+i \widehat{\xi}^{2} & =\frac{a}{\bar{a}}\left(\xi^{1}+i \xi^{2}\right) .
\end{aligned}
$$

Since $\frac{a}{\bar{a}}$, can be written as $e^{i \varphi}$, we have a spatial rotation in the $(1,2)$ plane. Then by identifying

$$
\left(1-\frac{v^{2}}{c^{2}}\right)^{-\frac{1}{2}}=\frac{1}{2}\left(a \bar{a}+a^{-1} \bar{a}^{-1}\right)
$$


we have the Lorentz transformation

$$
\begin{aligned}
& \widehat{\xi}^{0}=\frac{\xi^{0}}{\left(1-\frac{v^{2}}{c^{2}}\right)^{\frac{1}{2}}}+\frac{\frac{v}{c} \xi^{3}}{\left(1-\frac{v^{2}}{c^{2}}\right)^{\frac{1}{2}}}, \\
& \widehat{\xi}^{3}=\frac{\xi^{3}}{\left(1-\frac{v^{2}}{c^{2}}\right)^{\frac{1}{2}}}+\frac{\frac{v}{c} \xi^{0}}{\left(1-\frac{v^{2}}{c^{2}}\right)^{\frac{1}{2}}} .
\end{aligned}
$$

We see that the special fractional linear transformation $\widehat{\zeta}=a^{2} \zeta$ corresponds to the standard Lorentz transformation with a spatial rotation.

Example 2 $\quad s=-1$ and $w=1$ coming from the $s=1$ and $w=-3$ isomorphism.

Applying $\widehat{\zeta}=a^{2} \zeta$ to the $s=-1, w=1$, case, e.g., to the invariant factor space of $\psi_{1}^{0} \equiv \psi_{1(1,-3)}^{0}=\psi_{1 i}^{0} Y_{1 i}^{1}+\psi_{1 i j}^{0} Y_{2 i j}^{1}+\ldots$

we have

$$
\begin{aligned}
\widehat{\eta}_{(-1,1)} & =\widehat{\psi}_{1 i}^{0} \bar{m}_{i}(\widehat{\zeta}, \overline{\widehat{\zeta}})=e^{-i \lambda} K \eta_{(-1,1)}=e^{-i \lambda} K \psi_{1 i}^{0}(\zeta, \bar{\zeta}) \\
\bar{m}_{a}(\zeta, \bar{\zeta}) & =\frac{\sqrt{2}}{2 P}\left(0,1-\zeta^{2},-i\left(1+\zeta^{2}\right), 2 \zeta\right)
\end{aligned}
$$

Comparing the coefficients of $\left(1, \zeta, \zeta^{2}\right)$, we find that

$$
\begin{aligned}
\widehat{\psi}_{1,1}^{0} & =\frac{1}{2}\left(a^{2}+a^{-2}\right) \psi_{1,1}^{0}-i \frac{1}{2}\left(a^{2}-a^{-2}\right) \psi_{1,2}^{0} \\
& =\frac{\psi_{1,1}^{0}}{\left(1-\frac{v^{2}}{c^{2}}\right)^{\frac{1}{2}}}-i \frac{\frac{v}{c} \psi_{1,2}^{0}}{\left(1-\frac{v^{2}}{c^{2}}\right)^{\frac{1}{2}}}, \\
\widehat{\psi}_{1,2}^{0} & =\frac{1}{2}\left[a^{-2}+a^{2}\right] \psi_{1,2}^{0}+\frac{1}{2} i\left[a^{2}-a^{-2}\right] \psi_{1,1}^{0} \\
& =\frac{\psi_{1,2}^{0}}{\left(1-\frac{v^{2}}{c^{2}}\right)^{\frac{1}{2}}}+i \frac{\frac{v}{c} \psi_{1,1}^{0}}{\left(1-\frac{v^{2}}{c^{2}}\right)^{\frac{1}{2}}}, \\
\widehat{\psi}_{1,3}^{0} & =\psi_{1,3}^{0} .
\end{aligned}
$$

If we had identified $\psi_{1 i}^{0}$ with a Maxwell field via

$$
\psi_{1 i}^{0}=\left(\psi_{1,1}^{0}, \psi_{1,2}^{0}, \psi_{1,3}^{0}\right)=(\mathbf{E}+i \mathbf{B})_{i},
$$

then Eq.127) would be equivalent to a Lorentz transformation of the Maxwell tensor $F^{a b}$. The six real components of $\psi_{1 i}^{0}$ thus corresponds to a skew-symmetric Lorentzian tensor [25].

\subsection{Products of Spin-s Harmonics}

For completeness we give several of the relevant Clebsch-Gordon products that were used. We have left out terms with $l$-values greater than two. 


$$
\begin{aligned}
& Y_{2 k l}^{2} Y_{2 i j}^{-2}=\frac{\delta_{i k} \delta_{j l}}{5}+i \frac{\sqrt{2}}{5} \delta_{j l} \epsilon_{i k e} Y_{1 e}^{0}-\frac{1}{7} \delta_{l j} Y_{2 i k}^{0}, \\
& Y_{2 k l}^{0} Y_{2 i j}^{0}=\frac{24}{5} \delta_{i k} \delta_{j l}+\frac{24}{7} \delta_{l j} Y_{2 i k}^{0}, \\
& Y_{2 k l}^{-1} Y_{2 i j}^{0}=-\frac{i 12 \sqrt{2}}{5} \delta_{i k} \epsilon_{l j f} Y_{1 f}^{-1}+\frac{12}{7} \delta_{l i} Y_{2 k j}^{-1} \text {, } \\
& Y_{2 k l}^{1} Y_{2 i j}^{0}=\frac{i 12 \sqrt{2}}{5} \delta_{i k} \epsilon_{l j f} Y_{1 f}^{1}+\frac{12}{7} \delta_{l i} Y_{2 k j}^{1} \text {, } \\
& Y_{1 i}^{1} Y_{1 j}^{-1}=\frac{1}{3} \delta_{i j}-\frac{i \sqrt{2}}{4} \epsilon_{i j k} Y_{1 k}^{0}-\frac{1}{12} Y_{2 i j}^{0}, \\
& Y_{2 i j}^{2} Y_{1 k}^{-1}=\frac{3}{5} \delta_{j k} Y_{1 i}^{1}-\frac{i \sqrt{2}}{6} \epsilon_{i k l} Y_{2 j l}^{1}, \quad \bullet Y_{2 i j}^{2} Y_{1 k}^{0}=\frac{i 2 \sqrt{2}}{3} \epsilon_{i k l} Y_{2 j l}^{2}, \\
& Y_{2 i j}^{2} Y_{2 m l}^{0}=-\frac{24}{7} \delta_{l j} Y_{2 i m}^{2}, \quad \bullet Y_{2 l m}^{2} Y_{2 i j}^{-1}=\frac{2 \sqrt{2}}{5} i \delta_{i m} \epsilon_{l j f} Y_{1 f}^{1}+\frac{6}{7} \delta_{l i} Y_{2 m j}^{1}, \\
& Y_{2 i j}^{-1} Y_{1 k}^{1}=\frac{3}{5} Y_{1 i}^{0} \delta_{j k}+\frac{i \sqrt{2}}{6} \epsilon_{j k l} Y_{2 i l}^{0}, \quad \quad \quad Y_{2 i j}^{1} Y_{1 k}^{-1}=\frac{3}{5} Y_{1 i}^{0} \delta_{j k}-\frac{i \sqrt{2}}{6} \epsilon_{j k l} Y_{2 i l}^{0} \text {, } \\
& Y_{1 k}^{1} Y_{1 i}^{0}=\frac{i}{\sqrt{2}} \epsilon_{k i l} Y_{1 l}^{1}+\frac{1}{2} Y_{2 k i}^{1}, \quad \bullet Y_{1 k}^{-1} Y_{1 i}^{0}=-\frac{i}{\sqrt{2}} \epsilon_{k i l} Y_{1 l}^{-1}+\frac{1}{2} Y_{2 k i}^{-1}, \\
& Y_{1 k}^{1} Y_{2 i j}^{0}=-\frac{6}{5} Y_{1 j}^{1} \delta_{i k}+i \sqrt{2} \epsilon_{k i l} Y_{2 j l}^{1}, \quad \bullet Y_{1 k}^{-1} Y_{2 i j}^{0}=-\frac{6}{5} Y_{1 j}^{-1} \delta_{i k}-i \sqrt{2} \epsilon_{k i l} Y_{2 j l}^{-1} \text {, } \\
& Y_{2 m l}^{1} Y_{1 i}^{0}=\frac{6}{5} Y_{1 l}^{1} \delta_{i m}-\frac{i}{3} \sqrt{2} \epsilon_{i m f} Y_{2 l f}^{1}, \quad \bullet Y_{2 k l}^{-1} Y_{1 i}^{0}=\frac{6}{5} Y_{1 l}^{-1} \delta_{i k}+\frac{i}{3} \sqrt{2} \epsilon_{i k f} Y_{2 l f}^{-1} \text {, } \\
& Y_{1 i}^{0} Y_{1 j}^{0}=\frac{2}{3} \delta_{i j}+\frac{1}{3} Y_{2 i j}^{0}, \quad \bullet Y_{1 i}^{0} Y_{2 j k}^{0}=\frac{12}{5} \delta_{i j} Y_{1 k}^{0},
\end{aligned}
$$

\subsection{Angular Momentum Ambiguities}

As we mentioned earlier in the text there have been ambiguities, described in the literature 4, in the definition of the asymptotic angular momentum, $J^{* k}$. In our notation, (omitting the Maxwell field), the ambiguities are in the arbitrary choice of the constant $p$ in the expression:

$$
J^{* k}=-\left.\frac{\sqrt{2} c^{3}}{12 G} \psi_{1 k}^{0}\right|_{I}+p \frac{c^{3}}{G} \operatorname{Im}\left[\sigma ð \bar{\sigma}+\frac{1}{2} ð(\sigma \bar{\sigma})\right]_{k} .
$$

The default choices appear to be either 2,1 , or 0 . The present work does not influence or help resolve the ambiguity since to second order the expression

$$
\operatorname{Im}\left[\sigma \partial \bar{\sigma}+\frac{1}{2} ð(\sigma \bar{\sigma})\right]_{k}=i \frac{3 \cdot(24)^{2} \sqrt{2}}{10}\left(\xi^{i l} \bar{\xi}^{i j}+\bar{\xi}^{i l} \xi^{i j}\right) \epsilon_{l j k},
$$

vanishes.

One might have thought that our flux law, (still omitting the Maxwell field), 


$$
\begin{aligned}
J^{k \prime} & =-\frac{(24)^{2} c^{3}}{5 G}\left(\xi_{R}^{i l} v_{R}^{i j}+\xi_{I}^{i l} v_{I}^{i j}\right) \epsilon_{l j k}, \\
J^{i} & =-\left.\frac{\sqrt{2} c^{3}}{12 G} \psi_{1 i}^{0}\right|_{I}
\end{aligned}
$$

would have an ambiguity in the flux, i.e., a total derivative, arising from the use of the chain-rule, e.g.,

$$
\epsilon_{l j k} \xi_{R}^{i l} v_{R}^{i j}=\epsilon_{l j k}\left(\xi_{R}^{i l} \xi_{R}^{i j}\right)^{\prime}-\epsilon_{l j k} v_{R}^{i l} \xi_{R}^{i j}=-\epsilon_{l j k} v_{R}^{i l} \xi_{R}^{i j} .
$$

This apparent ambiguity disappears since these total derivatives are equivalent to the expression in Eq. (133) and again vanish identically .

It is possible that if our calculations were repeated, but done to third order, the ambiguities could be resolved. 